\title{
Lattice determination of Sigma-Lambda mixing
}

\author{
R. Horsley, ${ }^{1}$ J. Najjar, ${ }^{2}$ Y. Nakamura ${ }^{3}$ H. Perltt, ${ }^{4}$ D. Pleiter, ${ }^{5}$ P. E. L. Rakow, ${ }^{6}$ G. Schierholz, ${ }^{7}$ \\ A. Schiller, ${ }^{4}$ H. Stüben, ${ }^{8}$ and J. M. Zanotti ${ }^{9}$ \\ (QCDSF-UKQCD Collaboration)
}

\author{
${ }^{1}$ School of Physics and Astronomy, University of Edinburgh, Edinburgh EH9 3FD, United Kingdom \\ ${ }^{2}$ Institut für Theoretische Physik, Universität Regensburg, 93040 Regensburg, Germany \\ ${ }^{3}$ RIKEN Advanced Institute for Computational Science, Kobe, Hyogo 650-0047, Japan \\ ${ }^{4}$ Institut für Theoretische Physik, Universität Leipzig, 04109 Leipzig, Germany \\ ${ }^{5}$ Jülich Supercomputer Centre, Forschungszentrum Jülich, 52425 Jülich, Germany \\ ${ }^{6}$ Theoretical Physics Division, Department of Mathematical Sciences, University of Liverpool, Liverpool \\ L69 3BX, United Kingdom \\ ${ }^{7}$ Deutsches Elektronen-Synchrotron DESY, 22603 Hamburg, Germany \\ ${ }^{8}$ Regionales Rechenzentrum, Universität Hamburg, 20146 Hamburg, Germany \\ ${ }^{9}$ Department of Physics, CSSM, University of Adelaide, Adelaide South Australia 5005, Australia
}

(Received 5 December 2014; published 14 April 2015)

\begin{abstract}
Isospin breaking effects in baryon octet (and decuplet) masses are due to a combination of up and down quark mass differences and electromagnetic effects and lead to small mass splittings. Between the Sigma and Lambda this mass splitting is much larger, this being mostly due to their different wave functions. However when isospin is broken, there is a mixing between these states. We describe the formalism necessary to determine the QCD mixing matrix and hence find the mixing angle and mass splitting between the Sigma and Lambda particles due to QCD effects.
\end{abstract}

DOI: 10.1103/PhysRevD.91.074512

PACS numbers: $12.38 . \mathrm{Gc}, 11.15 . \mathrm{Ha}, 12.38 .-\mathrm{t}$

\section{INTRODUCTION}

Mass breaking effects in hadron octets (and decuplets) are mainly due to a combination of quark mass differences and electromagnetic effects, but can also sometimes have an additional component due to mixing between the hadron states. In this article we consider the baryon octet as shown in Fig. 1 where the spin $\frac{1}{2}$ baryons are plotted in the $I_{3}-Y$ plane. The particles on the (outer) ring, namely the $n(d d u), p(u u d)$, $\Sigma^{-}(d d s), \Sigma^{+}(u u s)$ and $\Xi^{-}(s s d), \Xi^{0}(s s u)$ all consist of combinations of $a a b$ quarks (where we use the notation of denoting a quark, $q$, by $a, b, \ldots$ which can be the up $u$, down $d$ or strange $s$ quark). $a$ here are the flavor doubly represented quarks, while $b$ is the flavor singly represented quark. For $u-d$ quark mass differences these isospin breaking effects are small. Examples for the lowest baryon octet are the $n-p$, $\Sigma^{-}-\Sigma^{+}$and $\Xi^{-}-\Xi^{0}$ mass differences. In [1] we investigated the hadronic QCD contribution to these isospin breaking splittings using lattice techniques. In this article we extend these results to the $\Sigma^{0}-\Lambda^{0}$ baryon octet masses. The method developed here for the $\Sigma^{0}-\Lambda^{0}$ mass splitting will automatically encompass the other splittings.

The $\Sigma^{0}$ and $\Lambda^{0}$ masses $^{1}$ are accurately known; from the Particle Data Group [2] we have

\footnotetext{
${ }^{1}$ We use $\Sigma$ to stand for the unmixed Sigma particle (pure isospin 1) and $\Sigma^{0}$ to denote the physical Sigma particle, with mixed isospin. Similarly $\Lambda$ denotes the pure isospin 0 state, and $\Lambda^{0}$ the physical Lambda particle.
}

$$
M_{\Sigma^{0}}^{\exp }=1.192642(24) \mathrm{GeV}, \quad M_{\Lambda^{0}}^{\exp }=1.115683(6) \mathrm{GeV},
$$

giving a mass splitting of

$$
\left(M_{\Sigma^{0}}-M_{\Lambda^{0}}\right)^{\exp }=76.959(23) \mathrm{MeV} .
$$

This is very much larger than the other mass splittings mentioned above, which are all of the order of a few $\mathrm{MeV}$. It is also more complicated than other mass splittings as while both baryons have the same quark content, namely $u, d, s$, most of the mass difference is due to their different wave functions. However there will also be additional mixing between these states. This will be apparent when we later consider $\Sigma\left(l l^{\prime} s\right)$ and $\Lambda\left(l l^{\prime} s\right)$ where $l$ and $l^{\prime}$ are distinct quarks, but mass degenerate, which already has this large mass splitting.

Understanding how this mixing works will be useful for understanding other mixing cases, such as $\eta-\eta^{\prime}$ or $\omega-\phi$ meson mixing, for which lattice simulations are considerably more difficult as there are computationally intensive disconnected terms in the correlation function to consider, [3-6]. In these latter cases a state at the center of the octet (the pure " $\eta_{8}$ " octet state) mixes with a further singlet state, " $\eta_{1}$." The case here of $\Sigma^{0}-\Lambda^{0}$ mixing is a little different as the particles have the same quantum numbers but now lie in the same octet (as shown in Fig. 1). In Fig. 2 we sketch the expected situation for the Lambda and Sigma hadrons, 


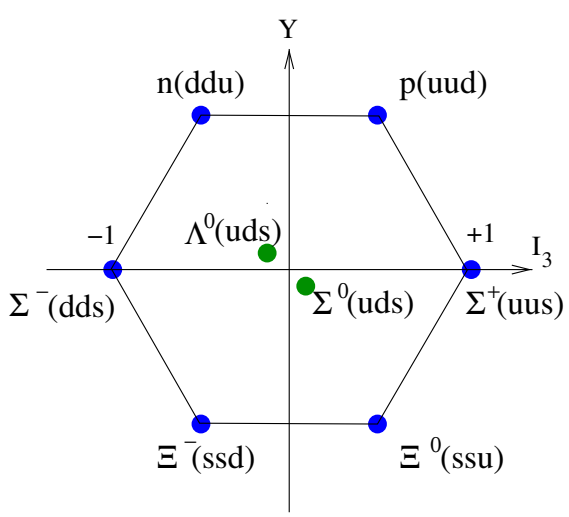

FIG. 1 (color online). The lowest octet for the spin $\frac{1}{2}$ baryons plotted in the $I_{3}-Y$ plane.

plotting $M_{B}^{2}$ against $m_{u}+m_{d}-2 m_{s}$. The lines represent lines of constant $m_{u}-m_{d}$, with the (red) dashed lines for $m_{u}-m_{d}=0$, while the (blue) lines are for $m_{u}-m_{d} \neq 0$. The central point is the quark mass symmetric point, when all quark masses are the same, when there is no difference between the Lambda and Sigma masses. In the isospin limit, when $m_{u}=m_{d} \neq m_{s}$ we sit at the points denoted by an open (red) circle. The mass splitting between the Sigma and Lambda particles is given by the vertical difference between these points.

However if $m_{u} \neq m_{d}$ then we have mixing between the Lambda and Sigma particles, as also depicted in the figure by (blue) lines. The physical $\Sigma^{0}$ and $\Lambda^{0}$ masses are now given by the (blue) filled circles. We see that there is then an additional mass splitting.

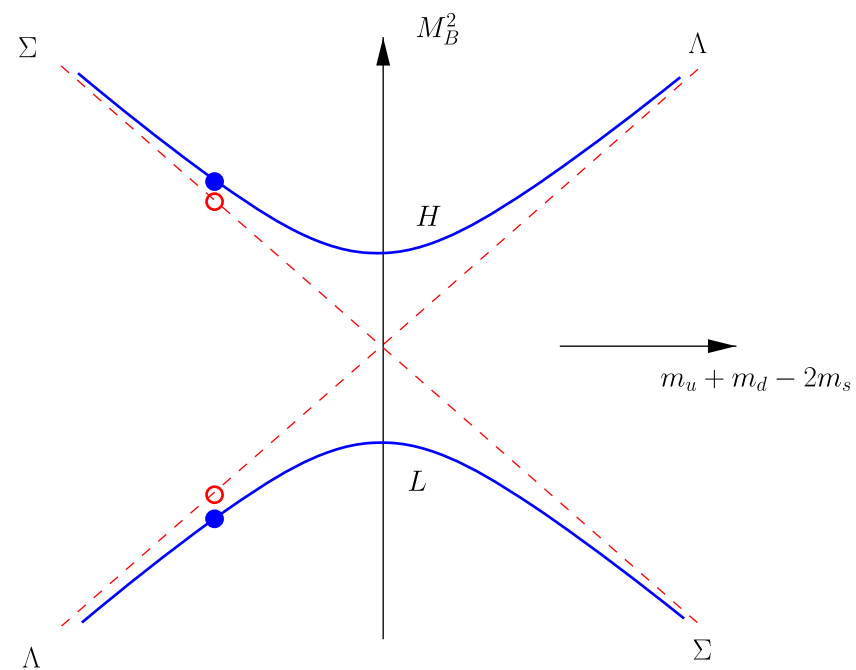

FIG. 2 (color online). A sketch of the heavy, $H$, and light, $L$, baryon (masses) ${ }^{2}$ against $m_{u}+m_{d}-2 m_{s}$ for fixed $m_{u}-m_{d}$. The mass splitting between the Sigma and Lambda masses in the isospin limit $\left(m_{u}=m_{d}\right)$ is given by the difference between the (red) circles; if $m_{u} \neq m_{d}$ then there is an additional mass difference due to mixing, the filled (blue) circles. Further explanation of the figure is given in the text.
As can also be seen from the figure, depending on the numerical values of the quark masses, the physical $\Sigma^{0}$ and $\Lambda^{0}$ masses can have a larger or smaller component of the original $\Sigma$ and $\Lambda$ particles. To avoid confusion we shall call in future the lower branch the light or $L$ branch with associated mass $M_{L}$, while the upper is the heavy or $H$ branch with mass $M_{H}$. For example in the isospin limit $m_{u}=m_{d} \equiv m_{l}$ we have

$M_{H}=\left\{\begin{array}{ll}M_{\Sigma} & m_{l}<m_{s} \\ M_{\Lambda} & m_{l}>m_{s}\end{array}, \quad M_{L}=\left\{\begin{array}{ll}M_{\Lambda} & m_{l}<m_{s} \\ M_{\Sigma} & m_{l}>m_{s}\end{array}\right.\right.$.

At the physical point, denoted by a $*$, we set

$$
M_{\Sigma^{0}}=M_{H}^{*}, \quad M_{\Lambda^{0}}=M_{L}^{*} .
$$

In the following we denote the pure octet, i.e. unmixed $\Sigma$ and $\Lambda$ mass states, by the Hermitian matrix

$$
\left(\begin{array}{ll}
M_{\Sigma \Sigma}^{2} & M_{\Sigma \Lambda}^{2} \\
M_{\Lambda \Sigma}^{2} & M_{\Lambda \Lambda}^{2}
\end{array}\right),
$$

while the mixed mass states will be denoted by $M_{H}^{2}, M_{L}^{2}$. We determine the mixing angle, $\theta_{\Sigma \Lambda}$, which rotates Eq. (5) with rotation matrix

$$
R=\left(\begin{array}{cc}
\cos \theta_{\Sigma \Lambda} & e^{i \phi_{\Sigma \Lambda}} \sin \theta_{\Sigma \Lambda} \\
-e^{-i \phi_{\Sigma \Lambda}} \sin \theta_{\Sigma \Lambda} & \cos \theta_{\Sigma \Lambda}
\end{array}\right),
$$

to the diagonal form

$$
\left(\begin{array}{cc}
M_{H}^{2} & 0 \\
0 & M_{L}^{2}
\end{array}\right)
$$

where $\phi_{\Sigma \Lambda}$ is the phase. Note that for the general symmetry arguments used here it does not matter whether they are applied to the hadron mass matrix, or some function of the mass matrix. We have chosen the quadratic form (see Sec. II A).

Although we discuss mixing between the $\Lambda$ and $\Sigma$ particles induced by quark mass differences, we neglect electromagnetic effects, which will also contribute mixing of roughly the same order of magnitude as isospin breaking effects. Thus we consider pure QCD effects only. The method also applies to mixing of $J^{P}=\frac{1}{2}^{+}$baryons in the singly charmed sector. For csu (or csd) baryons the hadronic mixing will be far larger than electromagnetic effects.

Previous determinations of $\Sigma-\Lambda$ mixing include using the quark model, e.g. [7], chiral perturbation theory, e.g. [8] and "sum rule" methods, e.g. $[9,10]$. 
The plan of this article is as follows. In the next section, Sec. II, we first discuss in more detail the calculational strategy that we employ here. In particular as summarized in Sec. II C, and discussed further in Appendixes A-B we make a $S U(3)$ flavor expansion about a point with degenerate mass $u, d$ and $s$ quarks. Section III then gives the $\Sigma-\Lambda$ mass mixing expansion up to next-to-leading order (NLO) (i.e. quadratic in the quark masses). We have actually computed the expansion to next-to-next-to-leading order (NNLO), but as we only use these to help to estimate systematic errors, the complete expansions are relegated to Appendix C. We also show numerical simulations with two mass degenerate sea quark masses as sufficient to determine the expansion coefficients also for the nondegenerate quark mass case. In Sec. IV we modify the expansion, to consider ratios, rather than lattice or scale dependent quantities. Some comments on matrix elements are given in Sec. V. Our numerical simulations are then detailed in Sec. VI and correlation functions and determination of the expansion coefficients are given in Secs. VIA-VIB, together with results for mass degenerate quarks. Finally our results and discussion are given in Sec. VII.

\section{THE $S U(3)$ FLAVOR EXPANSION}

\section{A. Mass matrix symmetries}

When all three quarks have the same mass, an $S U(3)$ transformation $U$ on the quark fields is a symmetry of the action; it leaves the quark mass matrix, $\mathcal{M}$, unchanged. We, however, are more interested in what happens in the case of unequal quark masses

$$
\mathcal{M}=\left(\begin{array}{ccc}
m_{u} & 0 & 0 \\
0 & m_{d} & 0 \\
0 & 0 & m_{s}
\end{array}\right),
$$

when we make an $S U(3)$ transformation

$$
\mathcal{M}^{\prime}=U \mathcal{M} U^{\dagger}
$$

Although this changes the quark matrix, it does not really change the physical situation. The eigenvalues of $\mathcal{M}^{\prime}$ are the same as those of $\mathcal{M}$, only the eigenvectors have been changed. Likewise, the mass spectrum of composite particles such as the mesons and baryons will not change; only the eigenvectors change.

This is easiest to see if the transformation $U$ is simply a permutation. For example, if we interchange $m_{d}$ and $m_{s}$ we still get the same set of baryon masses (see Fig. 1); all that changes is the names we give them. In this case, $M_{n}$ and $M_{\Xi^{0}}$ would be interchanged, as would $M_{p}$ and $M_{\Xi^{+}}$and so on. A rotation of the quark mass matrix simply leads to a corresponding rotation of the baryon mass matrix, $M$,

$$
M\left(U \mathcal{M} U^{\dagger}\right)=U M(\mathcal{M}) U^{\dagger}
$$

The $U$ matrices in Eq. (9) belong to a $3 \times 3$ matrix representation of $S U(3)$, while the $U$ matrices in Eq. (10) belong to an $8 \times 8$ representation of the same group.

We can see from Eq. (10) that the mass matrix and the (mass matrix) $)^{2}$ both transform in the same way,

$$
M^{2} \rightarrow\left(U M U^{\dagger}\right)\left(U M U^{\dagger}\right)=U M^{2} U^{\dagger}
$$

(where, as always, $M^{2}$ is shorthand for $M M$ ). Therefore, as far as symmetry arguments go, it makes no difference whether we discuss the hadron mass matrix, or the masssquared matrix. Note also that we can see from Eq. (11) that the eigenvectors of $M$ and of $M^{2}$ are the same.

We consider in future the $S U(3)$ flavor breaking expansion of $M^{2}$ rather than $M$, [8]. Thus we set

$$
M^{2}=\left(\begin{array}{cccccccc}
M_{n}^{2} & 0 & 0 & 0 & 0 & 0 & 0 & 0 \\
0 & M_{p}^{2} & 0 & 0 & 0 & 0 & 0 & 0 \\
0 & 0 & M_{\Sigma^{-}}^{2} & 0 & 0 & 0 & 0 & 0 \\
0 & 0 & 0 & M_{\Sigma \Sigma}^{2} & M_{\Sigma \Lambda}^{2} & 0 & 0 & 0 \\
0 & 0 & 0 & M_{\Lambda \Sigma}^{2} & M_{\Lambda \Lambda}^{2} & 0 & 0 & 0 \\
0 & 0 & 0 & 0 & 0 & M_{\Sigma^{+}}^{2} & 0 & 0 \\
0 & 0 & 0 & 0 & 0 & 0 & M_{\Xi^{-}}^{2} & 0 \\
0 & 0 & 0 & 0 & 0 & 0 & 0 & M_{\Xi^{0}}^{2}
\end{array}\right) .
$$

The reason is that as in [1] we have found again that better numerical fits in the quark mass range considered are obtained using the hadron mass matrix squared.

In Appendix A an explicit example for the transformation $u \leftrightarrow d$ is given.

\section{B. The $\Sigma-\Lambda$ mass matrix}

\section{Derivation}

The $S U(3)$ flavor expansion classifies mass polynomials according to the $S_{3}$ permutation group and the $S U(3)$ flavor group. $S_{3}$ is the symmetry group of an equilateral triangle, $C_{3 v}$. This group has three irreducible representations, [11], two different singlets, $A_{1}$ and $A_{2}$ and a doublet $E$, with elements $E^{+}$and $E^{-}$. Some details of this group and its representations are given in Appendix A of [12].

In [12] we classified the ten matrices $\left(N_{i}, i=1, \ldots, 10\right)$ which can contribute to the octet baryon mass matrix Eq. (12) according to their permutation, $S_{3}$ and $S U(3)$ symmetry; see Table I. The compact notation of Table I gives just the diagonal elements (the rows/columns being denoted by $n, p, \ldots)$. From Table I we see that seven of the 
TABLE I. Mass matrix contributions for octet baryons, classified by permutation and $S U(3)$ symmetry. (See Table V in [12].)

\begin{tabular}{|c|c|c|c|c|c|c|c|c|c|}
\hline$n$ & $p$ & $\Sigma^{-}$ & $\Sigma$ & $\Lambda$ & $\Sigma^{+}$ & $\Xi^{-}$ & $\Xi^{0}$ & $S_{3}$ & $S U(3)$ \\
\hline 1 & 1 & 1 & 1 & 1 & 1 & 1 & 1 & $A_{1}$ & 1 \\
\hline-1 & -1 & 0 & 0 & 0 & 0 & 1 & 1 & $E^{+}$ & $8_{a}$ \\
\hline-1 & 1 & -2 & 0 & 0 & 2 & -1 & 1 & $E^{-}$ & $8_{a}$ \\
\hline 1 & 1 & -2 & -2 & 2 & -2 & 1 & 1 & $E^{+}$ & $8_{b}$ \\
\hline-1 & 1 & 0 & \multicolumn{2}{|c|}{$\operatorname{mix}$} & 0 & 1 & -1 & $E^{-}$ & $8_{b}$ \\
\hline 1 & 1 & 1 & -3 & -3 & 1 & 1 & 1 & $A_{1}$ & 27 \\
\hline 1 & 1 & -2 & 3 & -3 & -2 & 1 & 1 & $E^{+}$ & 27 \\
\hline-1 & 1 & 0 & \multicolumn{2}{|c|}{$\operatorname{mix}$} & 0 & 1 & -1 & $E^{-}$ & 27 \\
\hline 1 & -1 & -1 & 0 & 0 & 1 & 1 & -1 & $A_{2}$ & $10, \overline{10}$ \\
\hline 0 & 0 & 0 & \multicolumn{2}{|c|}{$\operatorname{mix}$} & 0 & 0 & 0 & $A_{2}$ & $10, \overline{10}$ \\
\hline
\end{tabular}

matrices are diagonal; they can be read off directly from the table. For example the first row gives the $8 \times 8$ matrix: $\operatorname{diag}(1,1,1,1,1,1,1,1)$. The table also contains three matrices which mix the $\Sigma$ and $\Lambda$, the fifth, eighth rows which mix at the quadratic quark mass level and the tenth row which mixes with the cubic terms. All the matrices are explicitly listed in Appendix B. Thus we write

$$
M^{2}=\sum_{i=1}^{10} K_{i} N_{i}
$$

where $K_{i}$ are some functions of the quark masses (to be determined).

We now need the three nondiagonal matrices in full. From Appendix B they are $N_{5}, N_{8}$ and $N_{10}$. We thus have

$$
E^{-} 8_{b} \quad\left(\begin{array}{cccccccc}
-1 & 0 & 0 & 0 & 0 & 0 & 0 & 0 \\
0 & 1 & 0 & 0 & 0 & 0 & 0 & 0 \\
0 & 0 & 0 & 0 & 0 & 0 & 0 & 0 \\
0 & 0 & 0 & 0 & \frac{2}{\sqrt{3}} & 0 & 0 & 0 \\
0 & 0 & 0 & \frac{2}{\sqrt{3}} & 0 & 0 & 0 & 0 \\
0 & 0 & 0 & 0 & 0 & 0 & 0 & 0 \\
0 & 0 & 0 & 0 & 0 & 0 & 1 & 0 \\
0 & 0 & 0 & 0 & 0 & 0 & 0 & -1
\end{array}\right)
$$

$$
\left(\begin{array}{cccccccc}
0 & 0 & 0 & 0 & 0 & 0 & 0 & 0 \\
0 & 0 & 0 & 0 & 0 & 0 & 0 & 0 \\
0 & 0 & 0 & 0 & 0 & 0 & 0 & 0 \\
0 & 0 & 0 & 0 & -i & 0 & 0 & 0 \\
0 & 0 & 0 & i & 0 & 0 & 0 & 0 \\
0 & 0 & 0 & 0 & 0 & 0 & 0 & 0 \\
0 & 0 & 0 & 0 & 0 & 0 & 0 & 0 \\
0 & 0 & 0 & 0 & 0 & 0 & 0 & 0
\end{array}\right) .
$$

We are now ready to write down the general form of the $\Sigma-\Lambda$ mass matrix. From Table I we see that the $A_{1}$ terms always make equal contributions to the $\Sigma$ and $\Lambda$; and the $E^{+}$ terms always make opposite contributions to the $\Sigma$ and $\Lambda$. From Eqs. (14)-(15) we see that $E^{-}$terms contribute a real symmetric mixing term, and from Eq. (16) that $A_{2}$ terms contribute an imaginary, antisymmetric mixing. The allowed form of the $\Sigma-\Lambda$ mass matrix Eq. (5) is therefore

$$
\begin{aligned}
\left(\begin{array}{ll}
M_{\Sigma \Sigma}^{2} & M_{\Sigma \Lambda}^{2} \\
M_{\Lambda \Sigma}^{2} & M_{\Lambda \Lambda}^{2}
\end{array}\right)= & P_{A_{1}}\left(\begin{array}{ll}
1 & 0 \\
0 & 1
\end{array}\right)+P_{E^{+}}\left(\begin{array}{cc}
1 & 0 \\
0 & -1
\end{array}\right) \\
& +P_{E^{-}}\left(\begin{array}{ll}
0 & 1 \\
1 & 0
\end{array}\right)+P_{A_{2}}\left(\begin{array}{cc}
0 & -i \\
i & 0
\end{array}\right),
\end{aligned}
$$

where $P_{G}$ means a function of the quark masses with the symmetry $G$ under the $S_{3}$ permutation group.

We can also give a permutation argument for Eq. (17). The $\Sigma$ and $\Lambda$ form an $E$ representation of the permutation group, with the pure $\Sigma$ even under $u \leftrightarrow d$ and the $\Lambda$ odd. If $m_{u} \neq m_{d}$ there will be mixing between these states. Because the $\Sigma$ and $\Lambda$ have opposite behaviors under $u \leftrightarrow d$ exchange, the mass matrix for the $\Sigma-\Lambda$ system must have the behavior

$$
\left(\begin{array}{cc}
\text { even } & \text { odd } \\
\text { odd } & \text { even }
\end{array}\right)
$$

under the operation $u \leftrightarrow d$. The possible symmetries of the terms in the mass matrix are given by

$$
E \otimes E=A_{1} \oplus E \oplus A_{2}
$$

The $A_{1}$ and the $E^{+}$member of the $E$ doublet are even under $u \leftrightarrow d$, so they must be responsible for the diagonal part of the mass matrix. The mixing terms in the mass matrix are odd, so they must come from $E^{-}$and $A_{2}$ expressions.

From the above discussion we note that the formalism includes the no-mixing case when $m_{u}=m_{d}$; we simply set 


$$
P_{E^{-}}=0, \quad P_{A_{2}}=0,
$$

and the upper component of Eq. (17), now in a diagonal form, gives the degenerate mass of the Sigma baryons: $\Sigma \equiv$ $\left(\Sigma^{-}, \Sigma^{0}, \Sigma^{+}\right)$[which upon interchanging the quarks also gives the other baryon masses on the outer ring $N \equiv(n, p)$, $\left.\Xi \equiv\left(\Xi^{-}, \Xi^{0}\right)\right]$, while the lower component gives $\Lambda$.

\section{Diagonalization}

We now diagonalize the $2 \times 2$ (mass matrix) $)^{2}$ of Eq. (17) giving eigenvalues

$$
\begin{aligned}
& M_{H}^{2}=P_{A_{1}}+\sqrt{P_{E^{+}}^{2}+P_{E^{-}}^{2}+P_{A_{2}}^{2}} \\
& M_{L}^{2}=P_{A_{1}}-\sqrt{P_{E^{+}}^{2}+P_{E^{-}}^{2}+P_{A_{2}}^{2}},
\end{aligned}
$$

while if the eigenvectors are written as

$e_{H}=\left(\begin{array}{c}\cos \theta_{\Sigma \Lambda} \\ e^{-i \phi_{\Sigma \Lambda}} \sin \theta_{\Sigma \Lambda}\end{array}\right), \quad e_{L}=\left(\begin{array}{c}-e^{i \phi_{\Sigma \Lambda}} \sin \theta_{\Sigma \Lambda} \\ \cos \theta_{\Sigma \Lambda}\end{array}\right)$,

[cf Eq. (6)] we have

$$
\tan 2 \theta_{\Sigma \Lambda}=\frac{\sqrt{P_{E^{-}}^{2}+P_{A_{2}}^{2}}}{P_{E^{+}}}, \quad \tan \phi_{\Sigma \Lambda}=\frac{P_{A_{2}}}{P_{E^{-}}},
$$

for the mixing angle, $\theta_{\Sigma \Lambda}$, and phase, $\phi_{\Sigma \Lambda}$. Note that Eq. (21) trivially gives the $H$ and $L$ masses and also the mass difference $M_{H}-M_{L}$.

Alternatively the $P_{G}$ coefficients have some nice links to the $H$ and $L$ masses. $P_{A_{1}}$ gives the average (mass) $)^{2}$

$$
\frac{1}{2}\left(M_{H}^{2}+M_{L}^{2}\right)=P_{A_{1}}
$$

while the other three coefficients contribute symmetrically to the splitting of the two states

$$
\frac{1}{2}\left(M_{H}^{2}-M_{L}^{2}\right)=\sqrt{P_{E^{+}}^{2}+P_{E^{-}}^{2}+P_{A_{2}}^{2}} .
$$

\section{The $S U(3)$ flavor expansion}

Our strategy, as discussed in detail in [12] is to start from a point in the quark mass plane with all three sea quark masses equal,

$$
m_{u}=m_{d}=m_{s} \equiv m_{0}
$$

and extrapolate towards the physical point, denoted by a star, *, keeping the average sea quark mass

$$
\bar{m}=\frac{1}{3}\left(m_{u}+m_{d}+m_{s}\right)
$$

constant at the value $m_{0}$. As we approach the physical point, the $u$ and $d$ quarks become lighter, but the $s$ quark becomes heavier. Pions are decreasing in mass, but $K$ and $\eta$ increase in mass as we approach the physical point. Keeping $\bar{m}$ constant greatly reduces the number of mass polynomials which can occur in Taylor expansions of physical quantities within an $S U(3)$ multiplet. As we are expanding about the symmetric point, it is useful to introduce the notation

$$
\delta m_{q} \equiv m_{q}-\bar{m}, \quad q=u, d, s .
$$

Note that it follows from the definition that

$$
\delta m_{u}+\delta m_{d}+\delta m_{s}=0,
$$

so we could eliminate one of the $\delta m_{q}$ s. However we often keep all three terms as we can then write some expressions in a more obviously symmetrical form.

We can also generalize the $S U(3)$ flavor expansion to the case when the mass of the valence quarks can be different to the mass of the sea quarks, i.e. we leave the "unitary line." We call this the partially quenched (PQ) case. To do this we introduce

$$
\delta \mu_{q}=\mu_{q}-\bar{m}, \quad q=u, d, s,
$$

where $\mu_{q}$ is the valence quark mass. In distinction to the sea

\begin{tabular}{|c|c|c|c|c|}
\hline Polynomial & $S_{3}$ & \multicolumn{3}{|c|}{$S U(3)$} \\
\hline 1 & $A_{1}$ & 1 & & \\
\hline$\delta \mu_{u}+\delta \mu_{d}+\delta \mu_{s}$ & $A_{1}$ & 1 & & \\
\hline $2 \delta \mu_{s}-\delta \mu_{u}-\delta \mu_{d}$ & $E^{+}$ & & 8 & \\
\hline$\delta \mu_{u}-\delta \mu_{d}$ & $E^{-}$ & & 8 & \\
\hline$\left(\delta \mu_{u}+\delta \mu_{d}+\delta \mu_{s}\right)^{2}$ & $A_{1}$ & 1 & & \\
\hline$\left(\delta \mu_{u}+\delta \mu_{d}+\delta \mu_{s}\right)\left(2 \delta \mu_{s}-\delta \mu_{u}-\delta \mu_{d}\right)$ & $E^{+}$ & & 8 & \\
\hline$\left(\delta \mu_{u}+\delta \mu_{d}+\delta \mu_{s}\right)\left(\delta \mu_{u}-\delta \mu_{d}\right)$ & $E^{-}$ & & 8 & \\
\hline$\left(\delta \mu_{s}-\delta \mu_{u}\right)^{2}+\left(\delta \mu_{s}-\delta \mu_{d}\right)^{2}+\left(\delta \mu_{u}-\delta \mu_{d}\right)^{2}$ & $A_{1}$ & 1 & & 27 \\
\hline$\left(\delta \mu_{s}-\delta \mu_{u}\right)^{2}+\left(\delta \mu_{s}-\delta \mu_{d}\right)^{2}-2\left(\delta \mu_{u}-\delta \mu_{d}\right)^{2}$ & $E^{+}$ & & 8 & 27 \\
\hline$\left(\delta \mu_{s}-\delta \mu_{u}\right)^{2}-\left(\delta \mu_{s}-\delta \mu_{d}\right)^{2}$ & $E^{-}$ & & 8 & 27 \\
\hline$\delta m_{u}^{2}+\delta m_{d}^{2}+\delta m_{s}^{2}$ & $A_{1}$ & 1 & & 27 \\
\hline
\end{tabular}
quarks there is no restriction of the form Eq. (29) on the values of the valence quark masses. We give our results in this slightly more general case and then specialize to the unitary case $\delta \mu_{q} \rightarrow \delta m_{q}$ and then to the physical point $\delta m_{q} \rightarrow \delta m_{q}^{*}$. This generalization will prove to be useful for the numerical determination of the $S U(3)$ expansion coefficients.

TABLE II. All the quark mass polynomials needed for partially quenched masses, classified by symmetry properties. The table includes entries up to $O\left(\delta \mu_{q}^{2}\right)$ (Table XIV of [12]). 
In the following we give $S U(3)$ flavor symmetry breaking expansions up to cubic terms in the quark's mass, i.e. to $O\left(\delta \mu_{q}^{3}\right)$ (in both the sea and valence quarks). We call this the NNLO. However practically we see that the cubic terms contribute a small amount, so we regard this order as mainly being a "control" on the NLO results (for which analytic results are also given). In Table II we give the results to NLO.

\section{THE $\Sigma-\Lambda$ MIXING MASS FORMULA}

\section{A. Expansion of the $P_{G}$ coefficients}

We now return to the evaluation of the $\Sigma-\Lambda$ mass matrix as discussed in Sec. II B and demand that under all $S U(3)$ transformations
$\mathcal{M} \rightarrow \mathcal{M}^{\prime}=U \mathcal{M} U^{\dagger} \Leftrightarrow M^{2}\left(\mathcal{M}^{\prime}\right)=U M^{2}(\mathcal{M}) U^{\dagger}$.

Physically there is no change, just a relabeling of the states. For example $m_{d} \leftrightarrow m_{s}$ is equivalent to relabeling $M_{n} \leftrightarrow M_{\Xi^{0}}, \ldots$

The most general form of the partially quenched octet baryon mass matrix, for $1+1+1$ valence and sea quarks, up to order $\delta \mu_{q}^{3}$, in the case where $\bar{m}$ is held constant can now be determined. In Appendix B we illustrate explicitly the computation to leading order (LO) of the $S U(3)$ flavor expansion and $\Sigma-\Lambda$ mixing. We find that the coefficients ${ }^{2}$ in the $\Sigma-\Lambda$ mixing matrix, Eq. (17) are

$$
\begin{aligned}
P_{A_{1}}= & M_{0}^{2}+3 A_{1} \delta \bar{\mu}+\frac{1}{6} B_{0}\left(\delta m_{u}^{2}+\delta m_{d}^{2}+\delta m_{s}^{2}\right)+B_{1}\left(\delta \mu_{u}^{2}+\delta \mu_{d}^{2}+\delta \mu_{s}^{2}\right) \\
& +\frac{1}{4}\left(B_{3}+B_{4}\right)\left[\left(\delta \mu_{s}-\delta \mu_{u}\right)^{2}+\left(\delta \mu_{s}-\delta \mu_{d}\right)^{2}+\left(\delta \mu_{u}-\delta \mu_{d}\right)^{2}\right]+C_{0} \delta m_{u} \delta m_{d} \delta m_{s}+3 C_{1} \delta \bar{\mu}\left(\delta m_{u}^{2}+\delta m_{d}^{2}+\delta m_{s}^{2}\right) \\
& -4\left(C_{5}+C_{7}\right) \delta \mu_{u} \delta \mu_{d} \delta \mu_{s}+\frac{1}{2} Q_{1}\left(\delta \mu_{s}+\delta \mu_{u}\right)\left(\delta \mu_{s}+\delta \mu_{d}\right)\left(\delta \mu_{u}+\delta \mu_{d}\right)+\frac{27}{4} Q_{2}\left(\delta \mu_{s}-\delta \bar{\mu}\right)\left(\delta \mu_{u}-\delta \bar{\mu}\right)\left(\delta \mu_{d}-\delta \bar{\mu}\right), \\
P_{E^{+}}= & \frac{3}{2} A_{2}\left(\delta \mu_{s}-\delta \bar{\mu}\right)+\frac{1}{2} B_{2}\left(2 \delta \mu_{s}^{2}-\delta \mu_{u}^{2}-\delta \mu_{d}^{2}\right)+\frac{1}{4}\left(B_{3}-B_{4}\right)\left[\left(\delta \mu_{s}-\delta \mu_{u}\right)^{2}+\left(\delta \mu_{s}-\delta \mu_{d}\right)^{2}-2\left(\delta \mu_{u}-\delta \mu_{d}\right)^{2}\right] \\
& +\frac{3}{2} C_{2}\left(\delta \mu_{s}-\delta \bar{\mu}\right)\left(\delta m_{u}^{2}+\delta m_{d}^{2}+\delta m_{s}^{2}\right)+6\left(C_{3}-C_{4}\right)\left(\delta \mu_{s}-\delta \bar{\mu}\right) \delta \bar{\mu}^{2} \\
& +\frac{1}{6} Q_{3}\left[\left(\delta \mu_{s}-\delta \mu_{u}\right)^{2}+\left(\delta \mu_{s}-\delta \mu_{d}\right)^{2}-2\left(\delta \mu_{u}-\delta \mu_{d}\right)^{2}\right] \delta \bar{\mu}+\frac{1}{8} Q_{4}\left(\delta \mu_{s}-\delta \bar{\mu}\right)\left(\delta \mu_{u}^{2}+\delta \mu_{d}^{2}+\delta \mu_{s}^{2}-3 \delta \bar{\mu}^{2}\right), \\
P_{E^{-}}= & \frac{\sqrt{3}}{2} A_{2}\left(\delta \mu_{d}-\delta \mu_{u}\right)+\frac{\sqrt{3}}{2} B_{2}\left(\delta \mu_{d}^{2}-\delta \mu_{u}^{2}\right)+\frac{\sqrt{3}}{4}\left(B_{3}-B_{4}\right)\left[\left(\delta \mu_{s}-\delta \mu_{d}\right)^{2}-\left(\delta \mu_{s}-\delta \mu_{u}\right)^{2}\right] \\
& +\frac{\sqrt{3}}{2} C_{2}\left(\delta \mu_{d}-\delta \mu_{u}\right)\left(\delta m_{u}^{2}+\delta m_{d}^{2}+\delta m_{s}^{2}\right)+2 \sqrt{3}\left(C_{3}-C_{4}\right)\left(\delta \mu_{d}-\delta \mu_{u}\right) \delta \bar{\mu}^{2} \\
& +\frac{1}{8 \sqrt{3}} Q_{4}\left(\delta \mu_{d}-\delta \mu_{u}\right)\left(\delta \mu_{u}^{2}+\delta \mu_{d}^{2}+\delta \mu_{s}^{2}-3 \delta \bar{\mu}^{2}\right)-\frac{\sqrt{3}}{2} Q_{3}\left(\delta \mu_{d}-\delta \mu_{u}\right)\left(\delta \mu_{s}-\delta \bar{\mu}\right) \delta \bar{\mu}, \\
P_{A_{2}}= & C_{9}\left(\delta \mu_{s}-\delta \mu_{u}\right)\left(\delta \mu_{s}-\delta \mu_{d}\right)\left(\delta \mu_{u}-\delta \mu_{d}\right),
\end{aligned}
$$

where

$$
\begin{aligned}
& Q_{1} \equiv 2 C_{3}+C_{5}+C_{7} \\
& Q_{2} \equiv C_{5}-C_{6}+C_{7}+C_{8} \\
& Q_{3} \equiv 4\left(C_{3}-C_{4}\right)+3\left(C_{5}-C_{7}\right) \\
& Q_{4} \equiv 2\left(C_{3}-C_{4}\right)+3\left(C_{5}-C_{7}\right)-9\left(C_{6}+C_{8}\right),
\end{aligned}
$$

and

$$
\delta \bar{\mu} \equiv \frac{1}{3}\left(\delta \mu_{u}+\delta \mu_{d}+\delta \mu_{s}\right) .
$$

We can check that all the polynomials that occur here are polynomials of the appropriate symmetry from Table II (i.e.
Table XIV of [12]), or linear combinations of those polynomials. For example for $E^{+}$we have written

$$
\begin{aligned}
\frac{1}{2}\left(2 \delta \mu_{s}^{2}-\delta \mu_{u}^{2}-\delta \mu_{d}^{2}\right) \\
=\frac{1}{3}\left(\delta \mu_{u}+\delta \mu_{d}+\delta \mu_{s}\right)\left(2 \delta \mu_{s}-\delta \mu_{u}-\delta \mu_{d}\right) \\
\quad+\frac{1}{6}\left(\left(\delta \mu_{s}-\delta \mu_{u}\right)^{2}+\left(\delta \mu_{s}-\delta \mu_{d}\right)^{2}-2\left(\delta \mu_{u}-\delta \mu_{d}\right)^{2}\right) .
\end{aligned}
$$

\footnotetext{
${ }^{2}$ Note that $A_{1}$ and $A_{2}$ are used both for the $S_{3}$ representation and the expansion coefficient. Hopefully this will cause no confusion in the following.
} 
$P_{E^{+}}$and $P_{E^{-}}$form a doublet, i.e. they are related by $S_{3}$ symmetry, and involve the same parameters.

Using these expansions Eqs. (21) and (23) now give the $H$ and $L$ masses, together with the mixing angle $\theta_{\Sigma \Lambda}$ and phase $\phi_{\Sigma \Lambda}$. Note that in the unitary limit $\delta \mu_{q} \rightarrow \delta m_{q}$ these expressions simplify greatly,

$$
\begin{aligned}
P_{A_{1}} & =M_{0}^{2}+\frac{1}{12}\left(2 B_{0}+12 B_{1}+9 B_{3}+9 B_{4}\right)\left(\delta m_{u}^{2}+\delta m_{d}^{2}+\delta m_{s}^{2}\right)+\frac{1}{4}\left(4 C_{0}-16 C_{5}-16 C_{7}-16 Q_{1}+27 Q_{2}\right) \delta m_{u} \delta m_{d} \delta m_{s} \\
P_{E^{+}} & =\frac{3}{2} A_{2} \delta m_{s}+\frac{1}{8}\left(2 B_{2}+3 B_{3}-3 B_{4}\right)\left[3 \delta m_{s}^{2}-\left(\delta m_{u}-\delta m_{d}\right)^{2}\right]+\frac{1}{8}\left(12 C_{2}+Q_{4}\right) \delta m_{s}\left(\delta m_{u}^{2}+\delta m_{d}^{2}+\delta m_{s}^{2}\right) \\
P_{E^{-}} & =\frac{\sqrt{3}}{2} A_{2}\left(\delta m_{d}-\delta m_{u}\right)+\frac{\sqrt{3}}{4}\left(2 B_{2}+3 B_{3}-3 B_{4}\right) \delta m_{s}\left(\delta m_{u}-\delta m_{d}\right)+\frac{1}{8 \sqrt{3}}\left(12 C_{2}+Q_{4}\right)\left(\delta m_{u}-\delta m_{d}\right)\left(\delta m_{u}^{2}+\delta m_{d}^{2}+\delta m_{s}^{2}\right) \\
P_{A_{2}} & =C_{9}\left(\delta m_{s}-\delta m_{u}\right)\left(\delta m_{s}-\delta m_{d}\right)\left(\delta m_{u}-\delta m_{d}\right) .
\end{aligned}
$$

\section{B. Mass formulas, octet hadrons, $2+1$ case}

Let us now consider the equal mass valence up and down quark limit, i.e.

$$
\delta \mu_{u}=\delta \mu_{d} \equiv \delta \mu_{l}
$$

then $P_{E^{-}}=0=P_{A_{2}}$ (and $P_{A_{1}}, P_{E^{+}}$simplify) which means that $\theta_{\Sigma \Lambda}=0$, i.e. there is no $\Sigma-\Lambda$ mixing; Eq. (17) is already diagonal and so

$$
M_{\Sigma}^{2}=P_{A_{1}}+P_{E^{+}}, \quad M_{\Lambda}^{2}=P_{A_{1}}-P_{E^{+}},
$$

with $^{3} A_{2}>0$.

However as there is now no mixing the mass formula must also automatically describe the $\Sigma^{+}, \Sigma^{-}$and hence all the outer baryons with flavor structure $a a b$, Eq. (12). Replacing $\delta \mu_{l}$ by $\delta \mu_{a}$ and $\delta \mu_{s}$ by $\delta \mu_{b}$ we find

$$
\begin{aligned}
M_{\Sigma}^{2}(a a b) \equiv & M_{\Sigma}^{2}\left(a a^{\prime} b\right) \\
= & M_{0}^{2}+A_{1}\left(2 \delta \mu_{a}+\delta \mu_{b}\right)+A_{2}\left(\delta \mu_{b}-\delta \mu_{a}\right)+\frac{1}{6} B_{0}\left(\delta m_{u}^{2}+\delta m_{d}^{2}+\delta m_{s}^{2}\right) \\
& +B_{1}\left(2 \delta \mu_{a}^{2}+\delta \mu_{b}^{2}\right)+B_{2}\left(\delta \mu_{b}^{2}-\delta \mu_{a}^{2}\right)+B_{3}\left(\delta \mu_{b}-\delta \mu_{a}\right)^{2}+C_{0} \delta m_{u} \delta m_{d} \delta m_{s} \\
& +\left[C_{1}\left(2 \delta \mu_{a}+\delta \mu_{b}\right)+C_{2}\left(\delta \mu_{b}-\delta \mu_{a}\right)\right]\left(\delta m_{u}^{2}+\delta m_{d}^{2}+\delta m_{s}^{2}\right) \\
& +C_{3}\left(\delta \mu_{a}+\delta \mu_{b}\right)^{3}+C_{4}\left(\delta \mu_{a}+\delta \mu_{b}\right)^{2}\left(\delta \mu_{a}-\delta \mu_{b}\right) \\
& +C_{5}\left(\delta \mu_{a}+\delta \mu_{b}\right)\left(\delta \mu_{a}-\delta \mu_{b}\right)^{2}+C_{6}\left(\delta \mu_{a}-\delta \mu_{b}\right)^{3}
\end{aligned}
$$

The notation used here is meant to indicate that $a, a^{\prime}$ (and $\left.a^{\prime \prime}\right)$ are distinct quarks (in the baryon wave function), but are mass degenerate, i.e. $\mu_{a}=\mu_{a^{\prime}}\left(=\mu_{a^{\prime \prime}}\right){ }^{4}$ This agrees with our previous results in [1] and [12] [and justifies the notation for the expansion coefficients of Eq. (32)]. The valence flavor structure of Eq. (39) then describes

${ }^{3}$ From Eq. (32) we have $P_{E^{+}}=\frac{3}{2} A_{2}\left(\delta \mu_{s}-\delta \bar{\mu}\right)+\cdots=$ $A_{2}\left(\delta \mu_{s}-\delta \mu_{l}\right)+\cdots$ From Eq. (3) (and Fig. 2), generalizing to PQ quarks, Eq. (30), we see that with $A_{2}>0$ if $\delta \mu_{l}<\delta \mu_{s}$ then $M_{H}$ describes $M_{\Sigma}$ while if $\delta \mu_{l}>\delta \mu_{s}$ then $M_{L}$ describes $M_{\Sigma}$. Hence $M_{\Sigma}^{2}$ is always given by $P_{A_{1}}+P_{E^{+}}$. Similarly $M_{\Lambda}^{2}$ is given by $P_{A_{1}}-P_{E^{+}}$.

${ }^{4}$ It should be clear from the context whether we are referring to the Sigma particle or collectively to a particle on the outer ring of the octet. A similar comment holds for the Lambda. the broken isospin case of $p \equiv \Sigma(u u d), n \equiv \Sigma(d d u)$, $\Sigma^{+} \equiv \Sigma(u u s), \quad \Sigma^{-} \equiv \Sigma(d d s), \quad$ and $\quad \Xi^{0}=\Sigma(s s u), \quad \Xi^{-}=$ $\Sigma(s s d)$ as well as the isospin degenerate $\Sigma^{0} \equiv \Sigma\left(l l^{\prime} s\right)$. Furthermore now that we have cubic terms present, the Coleman-Glashow mass relation [13] is violated,

$$
\begin{aligned}
& M_{n}^{2}-M_{p}^{2}-M_{\Sigma^{-}}^{2}+M_{\Sigma^{+}}^{2}+M_{\Xi^{-}}^{2}-M_{\Xi^{0}}^{2} \\
& \quad=2\left(C_{4}-3 C_{6}\right)\left(\delta \mu_{s}-\delta \mu_{u}\right)\left(\delta \mu_{s}-\delta \mu_{d}\right)\left(\delta \mu_{d}-\delta \mu_{u}\right)
\end{aligned}
$$

(compare with Eq. (38) in [12]).

At the cubic level we have four new coefficients $C_{3}, C_{4}$, $C_{5}, C_{6}$ involving the valence quarks alone, and three new coefficients $C_{0}, C_{1}, C_{2}$ which involve the sea quark masses, and which drop out for calculations on the symmetric background, $\delta m_{q}=0$. Equation (39) assumes that $\bar{m}$, the 
average sea quark mass, is held constant. A large number of additional terms appear if that constraint is relaxed. If we work on single background all the sea quark terms can be absorbed into the valence parameters; the $B_{0}$ and $C_{0}$ terms can be absorbed into $M_{0}^{2} ; C_{1}$ and $C_{2}$ can be absorbed into $A_{1}$ and $A_{2}$ respectively.

Useful for numerical simulations is to take mass degenerate $u$ and $d$ sea quarks, so we have $2+1$ flavors rather than $1+1+1$ in the generation of configurations

$$
\delta m_{u}=\delta m_{d} \equiv \delta m_{l}
$$

which [together with Eq. (29)] is equivalent to the replacements

$$
\delta m_{l}^{2} \leftrightarrow \frac{1}{6}\left(\delta m_{u}^{2}+\delta m_{d}^{2}+\delta m_{s}^{2}\right), \quad \delta m_{l}^{3} \leftrightarrow-\frac{1}{2} \delta m_{u} \delta m_{d} \delta m_{s},
$$

in Eq. (32) for $P_{G}, G=A_{1}, E^{+}, E^{-}, A_{2}$.

Similarly we can write down the mass of the octet Lambda baryon as

$$
\begin{aligned}
M_{\Lambda}^{2}\left(a a^{\prime} b\right)= & M_{0}^{2}+A_{1}\left(2 \delta \mu_{a}+\delta \mu_{b}\right)-A_{2}\left(\delta \mu_{b}-\delta \mu_{a}\right)+\frac{1}{6} B_{0}\left(\delta m_{u}^{2}+\delta m_{d}^{2}+\delta m_{s}^{2}\right) \\
& +B_{1}\left(2 \delta \mu_{a}^{2}+\delta \mu_{b}^{2}\right)-B_{2}\left(\delta \mu_{b}^{2}-\delta \mu_{a}^{2}\right)+B_{4}\left(\delta \mu_{b}-\delta \mu_{a}\right)^{2}+C_{0} \delta m_{u} \delta m_{d} \delta m_{s} \\
& +\left[C_{1}\left(2 \delta \mu_{a}+\delta \mu_{b}\right)-C_{2}\left(\delta \mu_{b}-\delta \mu_{a}\right)\right]\left(\delta m_{u}^{2}+\delta m_{d}^{2}+\delta m_{s}^{2}\right) \\
& +C_{3}\left(\delta \mu_{a}+\delta \mu_{b}\right)^{3}+\left(C_{4}-2 C_{3}\right)\left(\delta \mu_{a}+\delta \mu_{b}\right)^{2}\left(\delta \mu_{b}-\delta \mu_{a}\right) \\
& +C_{7}\left(\delta \mu_{a}+\delta \mu_{b}\right)\left(\delta \mu_{b}-\delta \mu_{a}\right)^{2}+C_{8}\left(\delta \mu_{b}-\delta \mu_{a}\right)^{3}
\end{aligned}
$$

If all three quark masses are the same then all the masses become degenerate,

$$
M_{\Sigma}^{2}\left(a a a^{\prime \prime}\right) \equiv M_{\Sigma}^{2}\left(a a^{\prime} a^{\prime \prime}\right) \equiv M_{\Lambda}^{2}\left(a a^{\prime} a^{\prime \prime}\right) .
$$

In addition, for mass degenerate valence up and down quarks, the expansion in Eq. (39) now incorporates not only the mass degenerate nucleons, $p, n \equiv \Sigma\left(l l l^{\prime \prime}\right)$ with mass $M_{N}=M_{\Sigma}\left(l l l^{\prime \prime}\right)$, the Sigmas, $\Sigma^{-}, \Sigma^{+} \equiv \Sigma(l l s)$ and $\Sigma^{0} \equiv$ $\Sigma\left(l l^{\prime} s\right)$ with mass $M_{\Sigma}=M_{\Sigma}(l l s)=M_{\Sigma}\left(l l^{\prime} s\right)$ and Xis, $\Xi^{-}, \Xi^{0} \equiv \Xi(s s l)$ with mass $M_{\Xi}=M_{\Sigma}(s s l)$, but can also be extended to incorporate the fictitious baryon, $N_{s}\left(s s s^{\prime \prime}\right)$ with mass $M_{\Sigma}\left(s s s^{\prime \prime}\right)$. Furthermore the expansion in Eq. (43) also incorporates not only the Lambda, $\Lambda \equiv$ $\Lambda\left(l l^{\prime} s\right)$ with mass $M_{\Lambda}=M_{\Lambda}\left(l l^{\prime} s\right)$, but can be extended to the fictitious baryon $\Lambda_{2 s l}\left(s s^{\prime} l\right)$ with mass $M_{\Lambda}\left(s s^{\prime} l\right)$. For three mass degenerate valence quarks, we see that this mass formula reduces to the previous formula $M_{\Lambda}\left(l l^{\prime} l^{\prime \prime}\right)=$ $M_{\Sigma}\left(l l l^{\prime \prime}\right)$ and $M_{\Lambda}\left(s s^{\prime} s^{\prime \prime}\right)=M_{\Sigma}\left(s s s^{\prime \prime}\right)$.

The $\Lambda$ mass formula involves several parameters $B_{4}, C_{7}$ and $C_{8}$ which do not appear in the other baryon masses. We can understand why some terms in $M_{\Lambda}^{2}$ are constrained by the other hadron masses, while others are independent. The partially quenched quantities

$\left(X_{N}^{2}\right)^{\mathrm{PQ}} \equiv \frac{1}{3}\left(M_{N}^{2}+M_{\Sigma}^{2}+M_{\Xi}^{2}\right), \quad\left(X_{\Lambda}^{2}\right)^{\mathrm{PQ}} \equiv \frac{1}{2}\left(M_{\Lambda}^{2}+M_{\Sigma}^{2}\right)$

agree with each other exactly if $\delta \mu_{s}=\delta \mu_{l}$ [unbroken valence $S U(3)$, Eq. (44)]. The quantity $\left(X_{\Lambda}^{2}\right)^{\mathrm{PQ}}-\left(X_{N}^{2}\right)^{\mathrm{PQ}}$ is a 27-plet, so it should be $O\left(\left(\delta \mu_{s}-\delta \mu_{l}\right)^{2}\right)$ if valence $S U(3)$ is broken. Therefore any terms in $M_{\Lambda}^{2}$ which survive, or vanish more slowly than $\left(\delta \mu_{s}-\delta \mu_{l}\right)^{2}$, as $\delta \mu_{s} \rightarrow \delta \mu_{l}$ are constrained by the other baryon masses; any terms which vanish like $\left(\delta \mu_{s}-\delta \mu_{l}\right)^{2}$ or faster can have new independent coefficients unconnected to the other baryon masses. The $B_{4}, C_{7}$ and $C_{8}$ terms are the only terms in $M_{\Lambda}^{2}$ which vanish fast enough as $\delta \mu_{s} \rightarrow \delta \mu_{l}$ to evade this $\left(X_{\Lambda}^{2}\right)^{\mathrm{PQ}} \rightarrow\left(X_{N}^{2}\right)^{\mathrm{PQ}}$ constraint, as from Eqs. (39) and (43) we find ${ }^{5}$

$$
\begin{aligned}
\left(X_{\Lambda}^{2}\right)^{\mathrm{PQ}}-\left(X_{N}^{2}\right)^{\mathrm{PQ}} & \\
= & \frac{1}{6}\left(3 B_{4}-B_{3}\right)\left(\delta \mu_{s}-\delta \mu_{l}\right)^{2} \\
& +\frac{1}{18}\left(3 C_{7}-C_{5}-9 C_{6}+9 C_{8}\right)\left(\delta \mu_{s}-\delta \mu_{l}\right)^{3} \\
& +\frac{1}{9}\left(3 C_{7}-6 C_{3}-C_{5}\right)\left(\delta \mu_{s}+2 \delta \mu_{l}\right)\left(\delta \mu_{s}-\delta \mu_{l}\right)^{2} .
\end{aligned}
$$

${ }^{5}$ For completeness, we also give here the result for $\left(X_{\Lambda}^{2}\right)^{\mathrm{PQ}}$ $\left(X_{N}^{2}\right)^{\mathrm{PQ}}$ in the full $1+1+1$ case, generalizing Eq. (46),

$$
\begin{aligned}
\left(X_{\Lambda}^{2}\right)^{\mathrm{PQ}}-\left(X_{N}^{2}\right)^{\mathrm{PQ}} \\
=\frac{1}{4}\left(3 B_{4}-B_{3}\right)\left(\delta \mu_{u}^{2}+\delta \mu_{d}^{2}+\delta \mu_{s}^{2}-3 \delta \bar{\mu}^{2}\right) \\
\quad+\frac{3}{4}\left(3 C_{7}-C_{5}-9 C_{6}+9 C_{8}\right)\left(\delta \mu_{s}-\delta \bar{\mu}\right)\left(\delta \mu_{u}-\delta \bar{\mu}\right)\left(\delta \mu_{d}-\delta \bar{\mu}\right) \\
\quad+\frac{1}{2}\left(3 C_{7}-6 C_{3}-C_{5}\right)\left(\delta \mu_{u}^{2}+\delta \mu_{d}^{2}+\delta \mu_{s}^{2}-3 \delta \bar{\mu}^{2}\right) \delta \bar{\mu},
\end{aligned}
$$

where $\delta \bar{\mu}$ is defined in Eq. (34). 
At the $O\left(\delta \mu_{q}^{2}\right)$ level all the coefficients $M_{0}^{2}, A_{i}, B_{i}$ occur in Eqs. (39) and (43), and so can be found from a $2+1$ flavor partially quenched calculation. (The coefficients are functions of $\bar{m}$, and so will not change from a $1+1+1$ simulation to a $2+1$ simulation provided that $\bar{m}$ is held constant.) This will no longer hold at $O\left(\delta \mu_{q}^{3}\right)$; to find $P_{A_{2}}$ we would need to measure the other $A_{2}$ mass combination, which is the Coleman-Glashow violation, Eq. (40) or (38) of [12], which requires $1+1+1$ valence quarks and a more general expansion than given in Eqs. (39) and (43), so we have introduced a new coefficient, $C_{9}$, here.

\section{SCALE INDEPENDENT QUANTITIES}

\section{A. Ratios}

We now restrict ourselves to giving results to NLO (which will be sufficient for our numerical determinations). For completeness the full NNLO expressions are given in Appendix C.

Numerically it is advantageous to consider scale independent quantities, as previously discussed and used in $[1,12]$. As stated in Sec. IIC flavor blind (or singlet) quantities are suitable to form both scale independent mass ratios and to determine the scale. We denote these quantities generically by $X_{S}$. One useful type can be considered as the "center of mass" of the multiplet. Thus for the baryon octet, one possibility is

$$
\begin{aligned}
X_{N}^{2} & =\frac{1}{6}\left(M_{p}^{2}+M_{n}^{2}+M_{\Sigma^{+}}^{2}+M_{\Sigma^{-}}^{2}+M_{\Xi^{0}}^{2}+M_{\Xi^{-}}^{2}\right) \\
& =M_{0}^{2}+\left(\frac{1}{6} B_{0}+B_{1}+B_{3}\right)\left(\delta m_{u}^{2}+\delta m_{d}^{2}+\delta m_{s}^{2}\right) .
\end{aligned}
$$

At the physical point this has the value [2]

$$
X_{N}^{\exp }=1.1610 \mathrm{GeV} .
$$

As discussed in [12] flavor blind quantities, due to the vanishing of the linear $\delta m_{q}$ terms [see Eq. (47)] remain almost constant as we approach the physical point, so $a_{N}=$ $\left(a_{N} X_{N}\right) / X_{N}^{\exp }$ determines the lattice spacing $a_{N}\left(\kappa_{0}\right)$, [12]. (We have introduced the $N$ subscript as we are using $X_{N}$ to set the scale.)

We shall in future consider for the baryon octet the dimensionless ratios

$$
\tilde{M}^{2} \equiv \frac{M^{2}}{X_{N}^{2}}, \ldots
$$

and we wish to rewrite Eq. (21) in the form

$$
\begin{aligned}
& \tilde{M}_{H}^{2}=\tilde{P}_{A_{1}}+\sqrt{\tilde{P}_{E^{+}}^{2}+\tilde{P}_{E^{-}}^{2}+\tilde{P}_{A_{2}}^{2}} \\
& \tilde{M}_{L}^{2}=\tilde{P}_{A_{1}}-\sqrt{\tilde{P}_{E^{+}}^{2}+\tilde{P}_{E^{-}}^{2}+\tilde{P}_{A_{2}}^{2},}
\end{aligned}
$$

and Eq. (23) as

$$
\tan 2 \theta_{\Sigma \Lambda}=\frac{\sqrt{\tilde{P}_{E^{-}}^{2}+\tilde{P}_{A_{2}}^{2}}}{\tilde{P}_{E^{+}}}, \quad \tan \phi_{\Sigma \Lambda}=\frac{\tilde{P}_{A_{2}}}{\tilde{P}_{E^{-}}},
$$

where

$$
P_{G} \rightarrow \tilde{P}_{G}=\frac{P_{G}}{X_{N}^{2}}, \quad G=A_{1}, E^{+}, E^{-}, A_{2} .
$$

This can be achieved by defining

$$
\tilde{A}_{i}=\frac{A_{i}}{M_{0}^{2}}, \quad \tilde{B}_{i}=\frac{B_{i}}{M_{0}^{2}},
$$

together with the replacement

$$
B_{0} \rightarrow \tilde{B}_{0}=-6 \frac{B_{1}+B_{3}}{M_{0}^{2}}=-6\left(\tilde{B}_{1}+\tilde{B}_{3}\right) .
$$

The $\tilde{P}_{G}, G=A_{1}, E^{+}, E^{-}, A_{2}$ scale independent flavor $S U(3)$ expansion coefficients are then given to NLO by

$$
\begin{aligned}
\tilde{P}_{A_{1}}= & +3 \tilde{A}_{1} \delta \bar{\mu} \\
& +\frac{1}{6} \tilde{B}_{0}\left(\delta m_{u}^{2}+\delta m_{d}^{2}+\delta m_{s}^{2}\right)+\tilde{B}_{1}\left(\delta \mu_{a}^{2}+\delta \mu_{b}^{2}+\delta \mu_{c}^{2}\right) \\
& +\frac{1}{4}\left(\tilde{B}_{3}+\tilde{B}_{4}\right)\left[\left(\delta \mu_{c}-\delta \mu_{a}\right)^{2}\right. \\
& \left.+\left(\delta \mu_{c}-\delta \mu_{b}\right)^{2}+\left(\delta \mu_{a}-\delta \mu_{b}\right)^{2}\right] \\
\tilde{P}_{E^{+}}= & \frac{3}{2} \tilde{A}_{2}\left(\delta \mu_{c}-\delta \bar{\mu}\right)+\frac{1}{2} \tilde{B}_{2}\left(2 \delta \mu_{c}^{2}-\delta \mu_{a}^{2}-\delta \mu_{b}^{2}\right) \\
& +\frac{1}{4}\left(\tilde{B}_{3}-\tilde{B}_{4}\right)\left[\left(\delta \mu_{c}-\delta \mu_{a}\right)^{2}\right. \\
& \left.+\left(\delta \mu_{c}-\delta \mu_{b}\right)^{2}-2\left(\delta \mu_{a}-\delta \mu_{b}\right)^{2}\right] \\
& \sqrt{3} \tilde{A}_{2}\left(\delta \mu_{b}-\delta \mu_{a}\right) \\
\tilde{P}_{E^{-}}= & \sqrt{3} \tilde{B}_{2}\left(\delta \mu_{b}^{2}-\delta \mu_{a}^{2}\right) \\
& +\frac{\sqrt{3}}{4}\left(\tilde{B}_{3}-\tilde{B}_{4}\right)\left[\left(\delta \mu_{c}-\delta \mu_{b}\right)^{2}-\left(\delta \mu_{c}-\delta \mu_{a}\right)^{2}\right] \\
\tilde{P}_{A_{2}}= & 0,
\end{aligned}
$$

and

$$
\delta \bar{\mu} \equiv \frac{1}{3}\left(\delta \mu_{a}+\delta \mu_{b}+\delta \mu_{c}\right)
$$


(where we have written the more general $\delta \mu_{a}, \delta \mu_{b}, \delta \mu_{c}$ rather than the previous $\delta \mu_{u}, \delta \mu_{d}, \delta \mu_{s}$ respectively).

Similarly the changes to the baryon masses for mass degenerate up and down quarks are relatively simple. For completeness we give the scale independent flavor $S U(3)$ expansions

$$
\begin{aligned}
\tilde{M}_{\Sigma}^{2}(a a b)= & 1+\tilde{A}_{1}\left(2 \delta \mu_{a}+\delta \mu_{b}\right)+\tilde{A}_{2}\left(\delta \mu_{b}-\delta \mu_{a}\right) \\
& +\tilde{B}_{0} \delta m_{l}^{2}+\tilde{B}_{1}\left(2 \delta \mu_{a}^{2}+\delta \mu_{b}^{2}\right) \\
& +\tilde{B}_{2}\left(\delta \mu_{b}^{2}-\delta \mu_{a}^{2}\right)+\tilde{B}_{3}\left(\delta \mu_{b}-\delta \mu_{a}\right)^{2}
\end{aligned}
$$

and

$$
\begin{aligned}
\tilde{M}_{\Lambda}^{2}\left(a a^{\prime} b\right)= & 1+\tilde{A}_{1}\left(2 \delta \mu_{a}+\delta \mu_{b}\right)-\tilde{A}_{2}\left(\delta \mu_{b}-\delta \mu_{a}\right) \\
& +\tilde{B}_{0} \delta m_{l}^{2}+\tilde{B}_{1}\left(2 \delta \mu_{a}^{2}+\delta \mu_{b}^{2}\right) \\
& -\tilde{B}_{2}\left(\delta \mu_{b}^{2}-\delta \mu_{a}^{2}\right)+\tilde{B}_{4}\left(\delta \mu_{b}-\delta \mu_{a}\right)^{2}
\end{aligned}
$$

where $\tilde{B}_{0}$ is given in Eq. (54).

\section{B. Analytic expressions}

Finally we analytically expand out Eqs. (50)-(51) to NLO. On the unitary line (which is all that we shall later need) this gives

$$
\begin{aligned}
\tan 2 \theta_{\Sigma \Lambda}= & \frac{\left(\delta m_{d}-\delta m_{u}\right)}{\sqrt{3} \delta m_{s}} \\
& \times\left[1-\frac{1}{3}\left(\frac{2 \tilde{B}_{2}+3 \tilde{B}_{3}-3 \tilde{B}_{4}}{\tilde{A}_{2}}\right)\right. \\
& \left.\times \frac{\left(\delta m_{s}-\delta m_{u}\right)\left(\delta m_{s}-\delta m_{d}\right)}{\delta m_{s}}\right],
\end{aligned}
$$

and for the sum and difference, after additionally expanding further in the masses [rather than (mass) ${ }^{2}$ ]

$$
\begin{aligned}
& \frac{1}{2}\left(\tilde{M}_{\Sigma^{0}}+\tilde{M}_{\Lambda^{0}}\right) \\
& \quad=1+\frac{1}{8}\left(-\tilde{B}_{3}+3 \tilde{B}_{4}-\frac{3}{2} \tilde{A}_{2}^{2}\right)\left(\delta m_{u}^{2}+\delta m_{d}^{2}+\delta m_{s}^{2}\right),
\end{aligned}
$$

and

$$
\begin{aligned}
\tilde{M}_{\Sigma^{0}}-\tilde{M}_{\Lambda^{0}}= & \sqrt{\frac{3}{2}} \tilde{A}_{2} \sqrt{\delta m_{u}^{2}+\delta m_{d}^{2}+\delta m_{s}^{2}} \\
& \times\left[1+\frac{3}{2}\left(\frac{2 \tilde{B}_{2}+3 \tilde{B}_{3}-3 \tilde{B}_{4}}{\tilde{A}_{2}}\right)\right. \\
& \left.\times \frac{\delta m_{u} \delta m_{d} \delta m_{s}}{\delta m_{u}^{2}+\delta m_{d}^{2}+\delta m_{s}^{2}}\right] .
\end{aligned}
$$

In the isospin limit, upon using Eq. (41) we again see that the mixing angle in Eq. (59) vanishes, but the $\Sigma-\Lambda$ mass difference in Eq. (61) still persists. Let us first examine the convergence of the series. If we expand in terms of the quark mass difference $\delta m_{d}-\delta m_{u}$ and, now generalizing Eq. (41) slightly, the average quark mass $\delta m_{l}$ where $\delta m_{l}$ is given by

$$
\delta m_{l}=\left(\delta m_{u}+\delta m_{d}\right) / 2,
$$

then

$$
\begin{aligned}
-\frac{\left(\delta m_{s}-\delta m_{u}\right)\left(\delta m_{s}-\delta m_{d}\right)}{3 \delta m_{s}} & =\frac{3}{2} \delta m_{l}+O\left(\left(\delta m_{d}-\delta m_{u}\right)^{2}\right), \\
\frac{3 \delta m_{u} \delta m_{d} \delta m_{s}}{2\left(\delta m_{u}^{2}+\delta m_{d}^{2}+\delta m_{s}^{2}\right)} & =\frac{1}{2} \delta m_{l}+O\left(\left(\delta m_{d}-\delta m_{u}\right)^{2}\right) .
\end{aligned}
$$

At (or close to) the physical point $\delta m_{d} \approx \delta m_{u}$ so that in the expansion of $\tan 2 \theta_{\Sigma \Lambda}$, as compared to $\tilde{M}_{\Sigma^{0}}-\tilde{M}_{\Lambda^{0}}$ the NLO term is a factor $\approx 3$ larger, and hence the convergence of the $S U(3)$ symmetry flavor breaking series is expected to be worse for the mixing angle than for the mass difference.

As an estimate of the contribution of isospin breaking to $\Sigma-\Lambda$ mass splitting we expand in the difference between the isospin breaking and isospin symmetric cases giving to $\mathrm{LO}$,

$$
\left(\tilde{M}_{\Sigma^{0}}-\tilde{M}_{\Lambda^{0}}\right)-\left.\left(\tilde{M}_{\Sigma}-\tilde{M}_{\Lambda}\right)\right|_{\delta m_{l}}=\frac{1}{8} \tilde{A}_{2} \frac{\left(\delta m_{d}-\delta m_{u}\right)^{2}}{\left|\delta m_{l}\right|} .
$$

Mass splitting formulas for the baryons on the outer ring were given in [1] [Eqs. (12)-(15)]. For example we have

$$
\begin{aligned}
\tilde{M}_{n}-\tilde{M}_{p} \equiv & \tilde{M}_{\Sigma}(d d u)-\tilde{M}_{\Sigma}(u u d) \\
= & \frac{1}{2}\left(\delta m_{d}-\delta m_{u}\right)\left[\tilde{A}_{1}-2 \tilde{A}_{2}\right. \\
& \left.+\left(2 \tilde{B}_{1}-4 \tilde{B}_{2}-\frac{3}{2} \tilde{A}_{1}^{2}+3 \tilde{A}_{1} \tilde{A}_{2}\right) \delta m_{l}\right] .
\end{aligned}
$$

There are several differences between the isospin splitting between $\Sigma^{0}-\Lambda^{0}$ and $n-p$. For $\Sigma-\Lambda$ mixing from Eq. (64) we see that the mass splitting starts quadratically in $\left(\delta m_{d}-\delta m_{d}\right)$ while from Eq. (65) for $n-p$ the splitting is linear. Furthermore from Eq. (61) we see that this difference depends principally on $\tilde{A}_{2}$ and not at all on $\tilde{A}_{1}$. [The $\tilde{A}_{1}$ term has canceled in the unitary limit in Eq. (36).] This is completely opposite to the mass splittings of the baryons on the outer ring, [1] and Eq. (65), which depend on $\tilde{A}_{1}$ as well as $\tilde{A}_{2}$. As $\tilde{A}_{1}$ is numerically found to 
be much larger the result is then dominated by this coefficient.

We use these expansions in our numerical determinations. While for the central values of $M_{\Sigma^{0}}-M_{\Lambda^{0}}$ and $M_{n}-$ $M_{p}, \ldots$ it matters little whether we use these expressions or directly use those in Sec. IVA, for the error (particularly of $M_{n}-M_{p}, \ldots$ but rather less so for $\left.M_{\Sigma^{0}}-M_{\Lambda^{0}}\right)$ the difference depending on just one or two coefficients leads to a better determination.

\section{MATRIX ELEMENTS}

While we are primarily interested in this article in masses, we now make a few comments here on matrix elements. We see from Eq. (64) that in masses isospin breaking effects are second order in $\delta m_{d}-\delta m_{u}$. However, if we look at transition amplitudes instead of masses, the effects of the mixing angle appear at first order in $\theta_{\Sigma \Lambda}$, i.e. at first order in $\delta m_{d}-\delta m_{u}$, making an experimental determination of the mixing angle much more feasible.

It was pointed out in [14] that the semileptonic decays $\Sigma^{-} \rightarrow \Lambda^{0} e \bar{\nu}$ and $\Sigma^{+} \rightarrow \Lambda^{0} e^{+} \nu$ are particularly sensitive to the $\Sigma-\Lambda$ mixing angle. In the absence of mixing we would have

$$
\begin{aligned}
\Sigma^{-} \rightarrow \Sigma & A & =\sqrt{2}\left(\gamma_{\mu}+F \gamma_{\mu} \gamma_{5}\right) V_{u d} \\
\Sigma^{+} \rightarrow \Sigma & A & =-\sqrt{2}\left(\gamma_{\mu}+F \gamma_{\mu} \gamma_{5}\right) V_{u d} \\
\Sigma^{-} \rightarrow \Lambda & A & =\sqrt{\frac{2}{3}} D \gamma_{\mu} \gamma_{5} V_{u d} \\
\Sigma^{+} \rightarrow \Lambda & A & =\sqrt{\frac{2}{3}} D \gamma_{\mu} \gamma_{5} V_{u d},
\end{aligned}
$$

where $A$ is the amplitude, $F$ and $D$ are the axial $S U(3)$ couplings and $V_{u d} \sim \cos \theta_{C}$ is the appropriate CKM matrix element. There are two important points to note about these amplitudes. First, the $\Sigma^{-} \rightarrow \Lambda$ amplitude is equal to the $\Sigma^{+} \rightarrow \Lambda$ amplitude, while the $\Sigma^{-} \rightarrow \Sigma$ has the opposite sign to the $\Sigma^{+} \rightarrow \Sigma$. Second, the $\Sigma \rightarrow \Lambda$ amplitudes are purely axial, while the $\Sigma \rightarrow \Sigma$ amplitudes have a large vector contribution.

If we now introduce mixing as defined in Eq. (22),

$$
\Lambda^{0}=-\sin \theta_{\Sigma \Lambda} \Sigma+\cos \theta_{\Sigma \Lambda} \Lambda
$$

we have

$$
\begin{array}{rl}
\Sigma^{+} \rightarrow \Lambda^{0} & A=\left\{\sqrt{2} \gamma_{\mu} \sin \theta_{\Sigma \Lambda}+\left(\sqrt{\frac{2}{3}} D \cos \theta_{\Sigma \Lambda}+\sqrt{2} F \sin \theta_{\Sigma \Lambda}\right) \gamma_{\mu} \gamma_{5}\right\} V_{u d} \\
\Sigma^{-} \rightarrow \Lambda^{0} & A=\left\{-\sqrt{2} \gamma_{\mu} \sin \theta_{\Sigma \Lambda}+\left(\sqrt{\frac{2}{3}} D \cos \theta_{\Sigma \Lambda}-\sqrt{2} F \sin \theta_{\Sigma \Lambda}\right) \gamma_{\mu} \gamma_{5}\right\} V_{u d},
\end{array}
$$

for the transition amplitudes to the physical (mixed) $\Lambda^{0}$.

We see two effects which might be experimentally measurable at levels of the order of several percent. First, the $\Sigma \rightarrow \Lambda$ amplitudes have acquired a small vector component, which should change the angular distributions of the decay products. Second, the interference between the $D$ and $F$ components of the amplitudes works in opposite directions in the two cases. After correcting for phase space differences, we should see that the total $\Sigma^{+} \rightarrow \Lambda^{0}$ decay rate is enhanced, while the $\Sigma^{-} \rightarrow \Lambda^{0}$ is suppressed by mixing. Both effects are first order in the mixing, and so first order in $m_{d}-m_{u}$, and so they should be much more significant than the effect of mixing on the hadron masses.

In principle mixing effects of this sort appear in all decays of the $\Sigma^{0}$ and $\Lambda^{0}$, and all decays with a $\Sigma^{0}$ or $\Lambda^{0}$ in the decay products. All the semileptonic decays effected by the $\Sigma-\Lambda$ mixing are also discussed in [14].

\section{DETERMINATION OF THE EXPANSION COEFFICIENTS}

From Eq. (17) we see that we need to find the $2 \times 2$ mass matrix. We see from Eqs. (59) and (61) that to determine
$\theta_{\Sigma \Lambda}, M_{\Sigma^{0}}, M_{\Lambda^{0}}$ to LO we need to find the $\tilde{A}_{2}$ coefficient (to NLO also $\tilde{B}_{2}, \tilde{B}_{3}$ and $\tilde{B}_{4}$ ). We also need, of course, $\delta m_{u}^{*}$, $\delta m_{d}^{*}, \delta m_{s}^{*}$, i.e. a determination of the physical point. As apparent from Sec. III B, they can in principle all be determined from $2+1$ simulations of the $\Sigma$ and $\Lambda$ masses. However we have in addition also determined the offdiagonal matrix elements of the $2 \times 2$ mass matrix Eq. (17) for some PQ quark masses with $\delta \mu_{a} \neq \delta \mu_{b} \neq \delta \mu_{c}$.

Numerical simulations have been performed using $n_{f}=$ $2+1 O(a)$ improved clover fermions [15] at $\beta=5.50$ and mainly on $32^{3} \times 64$ lattice sizes, [12]. Errors given here are statistical [using $\sim O(1500)$ configurations] later together with an estimate of the systematic errors.

Once the $S U(3)$ flavor degenerate sea quark mass, $m_{0}$, is chosen, subsequent sea quark mass points $m_{l}, m_{s}$ are then arranged in the various simulations to keep $\bar{m}\left(=m_{0}\right)$ constant. This ensures that all the expansion coefficients given previously do not change. In [12] it was seen that a linear fit provides a good description of the numerical data on the unitary line over the relatively short distance from the $S U(3)$ flavor symmetric point down to the physical pion mass. This proved useful in helping us in choosing the 
initial point on the $S U(3)$ flavor symmetric line to give a path that reaches (or is very close to) the physical point.

The bare quark masses (both valence $\mu_{q}$ and unitary $\mu_{q} \rightarrow m_{q}$ ) in lattice units are given by

$$
\mu_{q}=\frac{1}{2}\left(\frac{1}{\kappa_{q}}-\frac{1}{\kappa_{0 c}}\right) \text { with } \quad q=l, s, 0, a, b,
$$

where vanishing of the quark mass along the $S U(3)$ flavor symmetric line determines $\kappa_{0 c}$. We denote the $S U(3)$ flavor symmetric kappa value, $\kappa_{0}$, as being the initial point on the path that leads to the physical point. This is given in Eq. (69) with $q=0$ and replacing $\mu_{0}$ by $m_{0}$. Keeping $\bar{m}=$ constant $=m_{0}$ then gives

$$
\delta \mu_{q}=\frac{1}{2}\left(\frac{1}{\kappa_{q}}-\frac{1}{\kappa_{0}}\right) .
$$

We see that $\kappa_{0 c}$ has dropped out of Eq. (70), so we do not need its explicit value here. While the choice of partially quenched quark masses is not restricted, along the unitary line the quark masses are restricted and we have

$$
\kappa_{s}=\frac{1}{\frac{3}{\kappa_{0}}-\frac{2}{\kappa_{l}}} .
$$

So a given $\kappa_{l}$ determines $\kappa_{s}$ here. The $S U(3)$ flavor symmetric $\kappa_{0}$ value chosen here for this action was found to be $\kappa_{0}=0.12090$ [12]. The constancy of flavor singlet quantities along the unitary line to the physical point [12] leads directly from $X_{N}$ to an estimation of the lattice spacing here of $a_{N}\left(\kappa_{0}=0.12090\right) \sim 0.079 \mathrm{fm}$.

\section{A. Correlation functions}

The wave functions (operators) used to determine the hadron masses are all taken to be Jacobi smeared. For the $\Sigma(a b c)$ and $\Lambda(a b c)$ we have

$$
\begin{aligned}
\mathcal{B}_{\Sigma(a b c) \alpha}(x)= & \frac{1}{\sqrt{2}} \epsilon^{a b c}\left(b_{\alpha}^{a}(x)\left[a^{b}(x)^{T_{D}} C \gamma_{5} c^{c}(x)\right]\right. \\
& \left.+a_{\alpha}^{a}(x)\left[b^{b}(x)^{T_{D}} C \gamma_{5} c^{c}(x)\right]\right), \\
\mathcal{B}_{\Lambda(a b c) \alpha}(x)= & \frac{1}{\sqrt{6}} \epsilon^{a b c}\left(2 c_{\alpha}^{a}(x)\left[a^{b}(x)^{T_{D}} C \gamma_{5} b^{c}(x)\right]\right. \\
& +b_{\alpha}^{a}(x)\left[a^{b}(x)^{T_{D}} C \gamma_{5} c^{c}(x)\right] \\
& \left.-a_{\alpha}^{a}(x)\left[b^{b}(x)^{T_{D}} C \gamma_{5} c^{c}(x)\right]\right),
\end{aligned}
$$

where $C=\gamma_{2} \gamma_{4}$ and the superscript ${ }^{T_{D}}$ denotes a transpose in Dirac space. ${ }^{6}$ The $\Sigma$ wave function is even under the interchange $a \leftrightarrow b$, while the $\Lambda$ wave function is odd under this interchange.

\footnotetext{
${ }^{6}$ The color indices are also denoted by $a, b$ and $c$; hopefully this will cause no confusion.
}

The correlation functions (on a lattice of temporal extension $T$ and spatial volume $V_{s}$ ) are given from the correlation matrix ${ }^{7}$

$$
\begin{aligned}
C_{i j}(t) & =\frac{1}{V_{s}} \operatorname{Tr}_{D} \Gamma_{\text {unpol }}\left\langle\sum_{\vec{y}} \mathcal{B}_{i}(\vec{y}, t) \sum_{\vec{x}} \overline{\mathcal{B}}_{j}(\vec{x}, 0)\right\rangle \\
& \propto A_{i} A_{j} e^{-M_{L} t}+B_{i} B_{j} e^{-M_{H} t}, \quad 0 \ll t \ll T / 2,
\end{aligned}
$$

with $i, j=\Sigma(a b c), \Lambda(a b c)$. This matrix is diagonalized, yielding $M_{H}$ and $M_{L}$.

As many of our choices of PQ valence quark masses have degenerate mass $a$ and $b$ quarks, as discussed in Sec. III B, some simplification for the $\Sigma$ wave function is possible. In this case we note that the Grassmann contractions lead to $C_{\Sigma\left(a a^{\prime} b\right) \Lambda\left(a a^{\prime} b\right)}=0$ identically, so that, as expected, the correlation matrix Eq. (73) is diagonal. Furthermore for the outer octet baryons we can use instead the wave function

$$
\mathcal{B}_{\Sigma(a a b) \alpha}(x)=\epsilon^{a b c} a_{\alpha}^{a}(x)\left[a^{b}(x)^{T_{D}} C \gamma_{5} b^{c}(x)\right]
$$

for valence quarks $a$ and $b$. The corresponding correlation function is

$$
\begin{aligned}
C_{\Sigma(a a b) \Sigma(a a b)}(t)= & \frac{1}{V_{s}} \operatorname{Tr}_{D} \Gamma_{\text {unpol }} \\
& \times\left\langle\sum_{\vec{y}} \mathcal{B}_{\Sigma(a a b)}(\vec{y}, t) \sum_{\vec{x}} \overline{\mathcal{B}}_{\Sigma(a a b)}(\vec{x}, 0)\right\rangle \\
& \propto A e^{-M_{\Sigma} t}, \quad 0 \ll t \ll T / 2 .
\end{aligned}
$$

This determines the $M_{\Sigma}(a a b)$ masses. Considering the correlation functions, $C_{\Sigma\left(a a^{\prime} b\right) \Sigma\left(a a^{\prime} b\right)}(t)$ and $C_{\Sigma(a a b) \Sigma(a a b)}(t)$, the Grassmann contractions can be shown to be equivalent so

$$
C_{\Sigma(a a b) \Sigma(a a b)}(t) \propto C_{\Sigma\left(a a^{\prime} b\right) \Sigma\left(a a^{\prime} b\right)}(t),
$$

and so the masses are the same, $M_{\Sigma(a a b)}=M_{\Sigma\left(a a^{\prime} b\right)}$, as indicated in in Eq. (39). Similarly when all the quark masses are degenerate, $C_{\Sigma\left(a a a^{\prime \prime}\right) \Sigma\left(a a a^{\prime \prime}\right)}(t) \propto$ $C_{\Lambda\left(a a^{\prime} a^{\prime \prime}\right) \Lambda\left(a a^{\prime} a^{\prime \prime}\right)}(t)$ or $M_{\Sigma\left(a a a^{\prime \prime}\right)}=M_{\Lambda\left(a a^{\prime} a^{\prime \prime}\right)}$ as expected.

Fitting to Eq. (50) then determines the $\tilde{A}, \tilde{B}$ coefficients. Together with a knowledge of the physical (and unitary) quark masses, $\delta m_{u}^{*}, \delta m_{d}^{*}, \delta m_{s}^{*}$, this leads to an evaluation of the physical $\Sigma^{0}$ and $\Lambda^{0}$ masses; see Eq. (4).

$$
{ }^{7} \Gamma_{\text {unpol }}=\frac{1}{2}\left(1+\gamma_{4}\right) .
$$




\section{B. Numerical results for the expansion coefficients}

Although simulations between the $S U(3)$ flavor symmetric point and the physical point are in principle enough to determine the expansion coefficients, in practice it is advantageous to increase the range to try to determine the NLO terms more reliably (i.e. with reduced error bars). However we also hope that the $S U(3)$ flavor breaking expansion developed here remains valid. As we see later in this section, for the $\Sigma-\Lambda$ splitting there is a further constraint. This leads to a choice of valence quark masses in the range $\left|\delta \mu_{a}\right|+\left|\delta \mu_{b}\right|+\left|\delta \mu_{c}\right| \lesssim 0.2$. This translates to nucleon masses of $\lesssim 2 \mathrm{GeV}$, so roughly the physical baryon masses lie in the middle of our fit range. The corresponding pion mass range is from about 800 down to $200 \mathrm{MeV}$, the $S U$ (3) flavor symmetric pion lying at about $420 \mathrm{MeV}$.

In order to determine these $\tilde{A}$ and $\tilde{B}$ coefficients, additional PQ masses have been determined on the set of gauge configurations that have all three sea quark masses equal, i.e. at the $S U(3)$ flavor symmetric point $\kappa_{0}=0.12090$. For these particular masses $\delta m_{l}=0=\delta m_{s}$ automatically. These masses are a mixture of masses with three distinctive valence quark masses (so we have mixing and $H$ and $L$ masses), together with two mass degenerate quark data, when there is no mixing. Thus we now make a simultaneous fit to Eq. (50) using the available data: the unitary data from [12] (the $32^{3} \times 64$ lattice data for $M_{N}$, $M_{\Lambda}, M_{\Sigma}, M_{\Xi}$ in Table XXII) together with some lighter quark mass data on a $48^{3} \times 96$ lattice. Specifically we have used 23 valence quark masses on the $32^{3} \times 64$ lattice with $\left(\kappa_{l}, \kappa_{s}\right)=(0.12090,0.12090)$, four valence quark masses on each of the $32^{3} \times 64$ lattice ensembles $\quad(0.12104,0.12062), \quad(0.121095,0.120512) \quad$ and $(0.121145,0.120413)$ and a further four on the $48^{3} \times 96$ lattice ensemble with $(0.121166,0.120371)$. All the fit data used are given in Appendix D.

There are two LO $(\tilde{A})$ and four NLO $(\tilde{B})$ coefficients to determine. Thus we have a six parameter fit for the fit functions in Eq. (50). It was found advantageous to preserve the identity of the $\Sigma$ and $\Lambda$ particles whenever possible, so for the mass degenerate PQ results, Eqs. (57)(58) were used. In Table III we give the results of this fit with bootstrap errors. With our normalization for the expansion coefficients all the numbers are $\sim O(10)$, except

TABLE III. Fit results for LO and NLO expansion coefficients.

\begin{tabular}{lr}
\hline \hline$\tilde{A}_{1}$ & $10.17(12)$ \\
$\tilde{A}_{2}$ & $1.849(124)$ \\
$\tilde{B}_{1}$ & $13.71(4.19)$ \\
$\tilde{B}_{2}$ & $-20.02(4.70)$ \\
$\tilde{B}_{3}$ & $-4.125(5.742)$ \\
$\tilde{B}_{4}$ & $-30.63(5.97)$ \\
\hline \hline
\end{tabular}

$\tilde{A}_{2}$ which is rather smaller. The (MINUIT) fit used gave $\chi^{2} /$ dof $\sim 38 / 60 \sim 0.6$ per degree of freedom.

Two simple plots which illustrate the fit results are first the completely mass degenerate case (when as discussed previously in Sec. VI A all outer baryon, $\Sigma$ and $\Lambda$ masses are the same), which may be illustrated by defining

$$
S_{\Sigma \Lambda} \equiv \tilde{M}_{\Sigma}^{2}\left(a a a^{\prime \prime}\right)=1+3 \tilde{A}_{1} \delta \mu_{a}+3 \tilde{B}_{1} \delta \mu_{a}^{2}
$$

Second we can consider the symmetric difference case (between $\Sigma$ and $\Lambda$ ) by setting

$$
\begin{aligned}
D_{\Sigma \Lambda}^{\mathrm{sym}} & \equiv \frac{\tilde{M}_{\Sigma}^{2}(a a b)-\tilde{M}_{\Lambda}^{2}\left(a a^{\prime} b\right)-\tilde{M}_{\Sigma}^{2}(b b a)+\tilde{M}_{\Lambda}^{2}\left(b b^{\prime} a\right)}{4\left(\delta \mu_{b}-\delta \mu_{a}\right)} \\
& =\tilde{A}_{2}+\tilde{B}_{2}\left(\delta \mu_{a}+\delta \mu_{b}\right) .
\end{aligned}
$$

(Again in these expressions and elsewhere $a^{\prime}, a^{\prime \prime}, \ldots$ are mass degenerate but distinct quarks.) At this order $D_{\Sigma \Lambda}^{\mathrm{sym}}$ is just a function of $\delta \mu_{a}+\delta \mu_{b}$; at higher orders [see Appendix C, Eq. (C7)] there are terms $\propto \delta \mu_{a}-\delta \mu_{b}$. Note that the choice for $D_{\Sigma \Lambda}^{\mathrm{sym}}$ tends to suppress them (and indeed eliminates them at NLO); this was the reason for the choice of this "symmetric derivative."

For the $S_{\Sigma \Lambda}$ we have the results shown in Fig. 3. For $S_{\Sigma \Lambda}$, the fit is very good and as indicated this could be easily extended to larger quark masses. As mentioned before $\tilde{A}_{1}$ is the relevant coefficient for mass splittings on the outer baryon ring.

In Fig. 4 we plot $D_{\Sigma \Lambda}^{\mathrm{sym}}$ against $\delta \mu_{a}+\delta \mu_{b}$. We see that the data are not linear in $\delta \mu_{a}+\delta \mu_{b}$. (As explained before we would not expect the data in this plot to lie on a unique

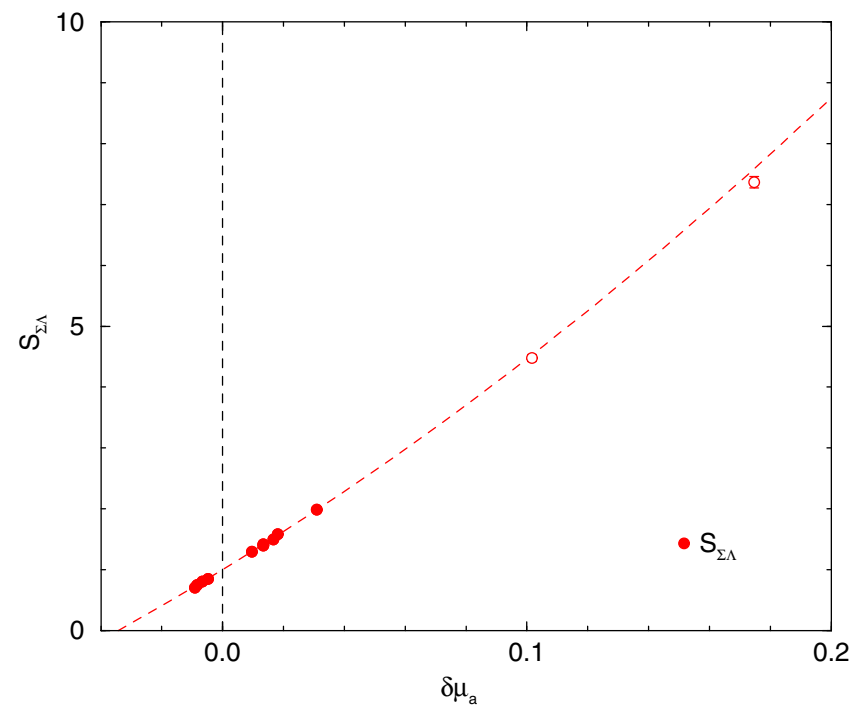

FIG. 3 (color online). $S_{\Sigma \Lambda}$ versus $\delta \mu_{a}\left[S_{\Sigma \Lambda}\right.$ is defined in Eq. (77)], together with a fit also given in Eq. (77). Points used in the fit are denoted by filled circles (those outside the fit range are given by open circles). 


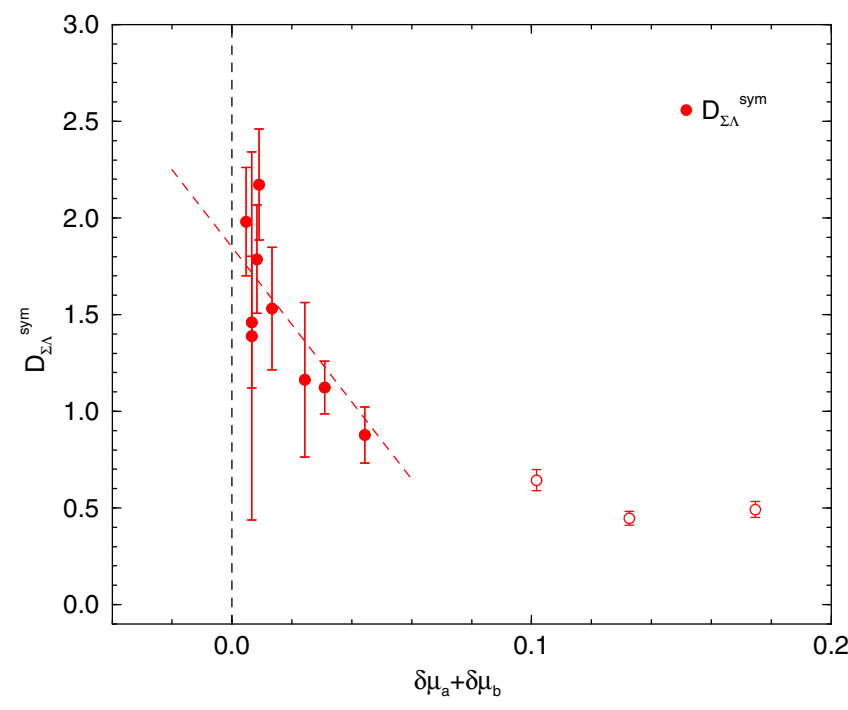

FIG. 4 (color online). $\quad D_{\Sigma \Lambda}^{\mathrm{sym}}$ versus $\delta \mu_{a}+\delta \mu_{b}\left[D_{\Sigma \Lambda}^{\mathrm{sym}}\right.$ is defined in Eq. (78)], together with the fit also given in Eq. (78). The same notation as in Fig. 3 is used.

curve due to the possible presence in the fit of terms proportional to $\delta \mu_{a}-\delta \mu_{b}$. However due to the choice of $D_{\Sigma \Lambda}^{\text {sym }}$ deviations should be small.) However despite this the plot has a sharp increase as the quark mass is reduced, indicating a possible nonpolynomial behavior there. As this is related to the $\Sigma-\Lambda$ mass splitting, this necessitates the restricted fit region, as compared to Fig. 3. (It should however also be noted that the unitary quark masses have $\left|\delta m_{a}\right| \lesssim 0.01$.)

The reason for this behavior is due to spin-spin interaction between the quarks. It is known (e.g. [16]) that in quark models the mass splittings are partially due to the QCD spin-spin interaction between the quarks. From the Dirac equation we know that the magnetic moment of a fermion $\propto 1 / m_{a}$, this holds in QCD too, for the chromomagnetic moment, which might suggest a spin-spin interaction of the form $\propto 1 /\left(m_{a} m_{b}\right)$. This has also recently been proposed in [17].

\section{The physical point}

Considering the equivalent pseudoscalar $S U(3)$ flavor breaking mass expansion as for the baryon octet and matching to the pseudoscalar meson masses gives $\delta m_{u}^{*}$, $\delta m_{d}^{*}, \delta m_{s}^{*}$. Again note that by considering the outer ring of the pseudoscalar octet, provided that the average quark mass $\bar{m}$ is held constant, the expansion coefficients can be determined from partially quenched $2+1$ flavor

TABLE IV. Results for the bare quark mass in lattice units at the physical point, slightly updated from [1].

\begin{tabular}{lcc}
\hline \hline$\delta m_{u}^{*}$ & $\delta m_{d}^{*}$ & $\delta m_{s}^{*}$ \\
\hline$-0.01140(3)$ & $-0.01067(3)$ & $0.02207(4)$ \\
\hline \hline
\end{tabular}

simulations rather than $1+1+1$ flavor expansions. This was discussed in [1] (and in particular the subtraction of QED effects) and we just quote the result of the analysis here, as given in Table IV. To cover uncertainties in electromagnetic effects arising from violations of Dashen's theorem, we assign a relative error $\sim 15 \%$ to the splitting $\delta m_{d}^{*}-\delta m_{u}^{*}$, [1].

\section{Comparison with "fan" plots}

We now compare the fit results with the mass values along the unitary line, i.e. which describe the evolution of the baryon masses along a path from the $S U(3)$ symmetric point down to the physical point in the isospin degenerate limit, i.e. $m_{u}=m_{d} \equiv m_{l}$. For this comparison we take the physical quark mass, in lattice units, from Table IV as

$$
\delta m_{l}^{*} \equiv\left(\delta m_{u}^{*}+\delta m_{d}^{*}\right) / 2=-0.01103(2) .
$$

In Fig. 5 we show the fan plot for all the $\Sigma$ - and $\Lambda$-type particles. We have $N\left(l l l^{\prime \prime}\right)\left[=\Lambda_{3 l}\left(l l^{\prime} l^{\prime \prime}\right)\right], \Lambda\left(l l^{\prime} s\right), \Sigma(l l s)$,

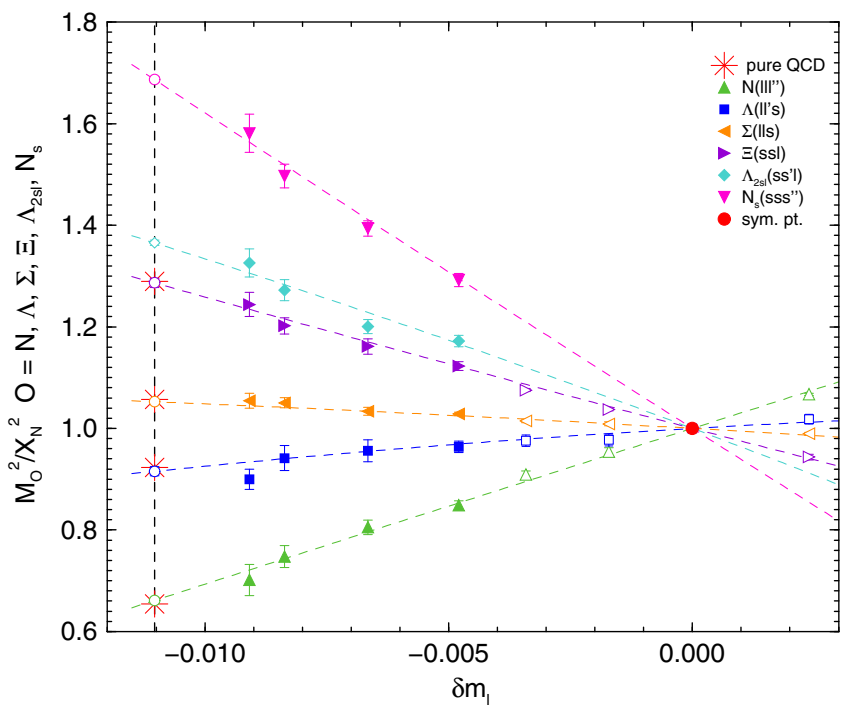

FIG. 5 (color online). The baryon fan plot for the $\Sigma$ - and $\Lambda$-type particles $\tilde{M}_{O}^{2}\left(O=N, \Lambda, \Sigma, \Xi, \Lambda_{2 s l}, N_{s}\right)$ versus $\delta m_{l}$. Filled up triangles, squares, left triangles, right triangles, diamonds and down triangles are the $N\left(l l l^{\prime \prime}\right), \Lambda\left(l l^{\prime} s\right), \Sigma(l l s), \Xi(s s l), \Lambda_{l 2 s}\left(s s^{\prime} l\right)$ and $N_{s}\left(s s s^{\prime \prime}\right)$ results respectively using $32^{3} \times 64$ sized lattices. The common symmetric point is the filled circle. The open up triangles, left triangles, right triangles and down triangles are from comparison $24^{3} \times 48$ sized lattices (and not used in the fits here). The vertical dashed line from Eq. (79) is the $n_{f}=2+1$ pure QCD physical point, with the open circles being the numerically determined pure QCD hadron mass ratios for $2+1$ quark flavors. For comparison, the stars represent the average of the (mass) $)^{2}$ of $M_{N}^{* 2}\left(l l l^{\prime \prime}\right)=\left(M_{n}^{\exp 2}(d d u)+\right.$ $\left.M_{p}^{\exp 2}(u u d)\right) / 2, \quad M_{\Lambda}^{* 2}(l l s)=M_{\Lambda^{0}}^{\exp 2}(u d s), \quad M_{\Sigma}^{* 2}(l l s)=$ $\left(M_{\Sigma^{-}}^{\exp 2}(d d s)+M_{\Sigma^{+}}^{\exp 2}(u u s)\right) / 2$ and $M_{\Xi}^{* 2}(s s l)=\left(M_{\Xi^{-}}^{\exp 2}(s s d)+\right.$ $\left.M_{\Xi^{0}}^{\exp 2}(s s u)\right) / 2$. 
$\Xi(s s l), \Lambda_{2 s l}\left(s s^{\prime} l\right)$ and $N_{s}\left(s s s^{\prime \prime}\right)\left[=\Lambda_{3 s}\left(s s^{\prime} s^{\prime \prime}\right] . \quad\left[N_{s}\left(s s s^{\prime \prime}\right)\right.\right.$ and $\Lambda_{2 s l}\left(s s^{\prime} l\right)$ are fictitious baryons, but provide additional useful data for the fits.] As this is the diagonal case there is no mixing and from Eq. (38) the fit is given by $\tilde{M}_{N}^{2}=P_{A_{1}}+P_{E^{+}}, \tilde{M}_{\Lambda}^{2}=P_{A_{1}}-P_{E^{+}}$. We find good agreement with the expected results.

It can easily be seen ("ruler test") that the fits are dominated by the LO in the $S U(3)$ flavor symmetry breaking expansion. Given the fit results, this is not so surprising, as for the unitary results we have a maximum quark mass given by $\left|\delta m_{l}\right| \sim 0.01$, which is rather small (certainly in comparison with many of the PQ masses used) and indicates that at least in the region we are interested in the low order $S U(3)$ flavor breaking expansion describes the data well.

For completeness we give here the values at the $2+1$ QCD physical point (open circles in Fig. 5) of $\tilde{M}_{N}^{* 2}=0.6612(58), \tilde{M}_{\Lambda}^{* 2}=0.9155(89), \tilde{M}_{\Sigma}^{* 2}=1.052(4)$, $\tilde{M}_{\Xi}^{* 2}=1.286(9), \tilde{M}_{\Lambda_{2 s l}}^{* 2}=1.365(5)$ and $\tilde{M}_{N_{s}}^{* 2}=1.687(6)$. For a comparison to these values, the stars in Fig. 5 represent the average of the squared experimental masses of the appropriate particles, as defined in the figure caption.

\section{RESULTS AND CONCLUSIONS}

We now give results for the QCD contribution to the baryon masses and their splittings.

\section{A. Outer ring of the baryon octet}

We first discuss the masses on the outer ring of the baryon octet using the physical quark masses given in Table IV and the expansion coefficients as given in Table III.

Possible sources of systematic errors are discussed in Appendix A of [1] as coming from the following: finite lattice volume, convergence of the $S U(3)$ flavor symmetry breaking expansion, the path to the physical point and finite lattice spacing (to which we refer the reader). As the data set used has not changed greatly, the systematic errors are little effected, so we use the same methods giving similar results as determined there. For the mass ratios $\tilde{M}$ we find estimates of systematic errors of $\sim 1 \%$ for finite volume, $\sim 1 \%$ for the flavor symmetry expansion (it is also apparent from Fig. 5 that in the region we are interested in curvature effects are very small), $\sim 4 \%$ as the chosen $\kappa_{0}$ and hence the trajectory in the $m_{s}-m_{l}$ plane does not quite go through the physical point, while the systematic errors arising from a finite lattice spacing are small.

We find the results for the masses and splittings of Table V. For the splittings, rather than using Eq. (50) directly (i.e. the results of the left panel of Table V) we use the expressions in Sec. IV B. As discussed there, for the central values it makes little difference; however the error is now better determined. For the baryons on the outer ring of the octet the central values (both for masses and mass
TABLE V. Left panel: Baryon masses on the outer ring of the octet. The second column gives the quark content, while the third column, Exp, gives the experimental masses from [2]. The last column, Result, gives the result from this work. The first error is the statistical error, while the second is the total systematic error (in quadrature). $X_{N}^{\exp }$ from Eq. (48) has been used to convert to $\mathrm{GeV}$. Right panel: Baryon mass splittings on the outer ring. The third error is due to possible violations in Dashen's theorem, Sec. VI C.

\begin{tabular}{|c|c|c|c|}
\hline \multicolumn{2}{|c|}{ Particle } & \multirow{2}{*}{$\frac{\operatorname{Exp}[\mathrm{GeV}]}{0.9383}$} & \multirow{2}{*}{$\frac{\text { Result }[\mathrm{GeV}]}{0.9427(41)(40)}$} \\
\hline$M_{p}$ & uud & & \\
\hline$M_{n}$ & $d d u$ & 0.9396 & $0.9454(40)(40)$ \\
\hline$M_{\Sigma^{+}}$ & uus & 1.1894 & $1.1874(23)(50)$ \\
\hline$M_{\Sigma^{-}}$ & $d d s$ & 1.1974 & $1.1947(22)(51)$ \\
\hline$M_{\Xi^{0}}$ & ssu & 1.3149 & $1.3145(49)(56)$ \\
\hline$M_{\Xi^{-}}$ & ssd & 1.3217 & $1.3191(48)(56)$ \\
\hline \multicolumn{2}{|c|}{ Splitting } & \multicolumn{2}{|c|}{ Result $[\mathrm{MeV}]$} \\
\hline \multirow{3}{*}{\multicolumn{2}{|c|}{$\begin{array}{l}M_{n}-M_{p} \\
M_{\Sigma^{-}}-M_{\Sigma^{+}} \\
M_{\Xi^{-}}-M_{\Xi^{0}}\end{array}$}} & \multicolumn{2}{|c|}{$2.70(15)(11)(40)$} \\
\hline & & \multicolumn{2}{|c|}{$7.27(22)(31)(109)$} \\
\hline & & \multicolumn{2}{|c|}{$4.57(19)(19)(68)$} \\
\hline
\end{tabular}

splittings) are in agreement with previous results, [1]. Note that we are not trying to compare the mass splittings with the experimental values, due to electromagnetic effects (not considered here).

\section{B. $\Sigma-\Lambda$ mixing}

We now turn to the result for $\Sigma-\Lambda$ mixing. In Table VI we give the $\Sigma^{0}$ and $\Lambda^{0}$ masses. The $\Sigma^{0}-\Lambda^{0}$ mass difference is

$$
M_{\Sigma^{0}}-M_{\Lambda^{0}}=79.44(7.37)(3.37) \mathrm{MeV} .
$$

(The same discussion for the determination of the errors as for the previous results, Sec. VII A, also holds here.) This is to be compared with the experimental result, Eq. (2) of 76.96(2) MeV. As both particles have the same quark content (and are uncharged) we do not expect much electromagnetic contribution. Between the LO and NLO result there is only a few percent difference. Furthermore taking the difference between the $M_{\Sigma^{0}}-M_{\Lambda^{0}}$ mass splitting in Table VI and $M_{\Sigma}^{*}(l l s)-M_{\Lambda}^{*}\left(l l^{\prime} s\right)$ (i.e. the isospin limit) gives a tiny contribution due to isospin breaking, consistent with zero and which our present results are not precise enough to reliably estimate.

TABLE VI. $\quad \Sigma^{0}$ and $\Lambda^{0}$ masses. The same notation is used as for the first panel of Table V.

\begin{tabular}{llll}
\hline \hline Particle & & Exp [GeV] & Result $[\mathrm{GeV}]$ \\
\hline$M_{\Sigma^{0}}$ & $u d s$ & 1.1926 & $1.1910(23)(51)$ \\
$M_{\Lambda^{0}}$ & $u d s$ & 1.1157 & $1.1109(54)(47)$ \\
\hline \hline
\end{tabular}


For the mixing angle we find

$$
\tan 2 \theta_{\Sigma \Lambda}=0.0123(45)(25),
$$

which, as anticipated, gives a very small angle, $\theta_{\Sigma \Lambda} \sim 0.006(3)$ rads $\lesssim 1^{\circ}$. Comparing with e.g. a quark model result [7] gives $\theta_{\Sigma \Lambda} \sim 0.01$ rads which is compatible with our result.

We note that the $\mathrm{LO}$ value of $\tan 2 \theta_{\Sigma \Lambda}$ from Eq. (59) is $\sim 0.0191$ so in this case with our determined $\tilde{A}$ and $\tilde{B}$ values for the $S U(3)$ flavor breaking expansion there is some reduction in the value of the angle when going to NLO. However in distinction to the $\Sigma^{0}-\Lambda^{0}$ mass difference the nonleading term is now much larger. This is because numerically $\quad\left(\delta m_{s}-\delta m_{u}\right)\left(\delta m_{s}-\delta m_{d}\right) /\left.3 \delta m_{s}\right|^{*} \sim 0.0166$ to be compared with $3 \delta m_{u} \delta m_{d} \delta m_{s} / 2\left(\delta m_{u}^{2}+\delta m_{d}^{2}+\right.$ $\left.\delta m_{s}^{2}\right)\left.\right|^{*} \sim 0.0056$, which as expected from the discussion in Sec. IV B is a factor 3 smaller. Thus the $S U(3)$ symmetry flavor breaking expansion for the mixing angle in Eq. (59) appears less convergent than for the $\Sigma^{0}-\Lambda^{0}$ mass difference, Eq. (61). In order to account for this, we have increased the relative systematic error associated with the flavor symmetry expansion to $\sim 15 \%$.

\section{CONCLUSIONS}

In this article we have extended our earlier work describing the QCD contribution to isospin breaking effects in baryon masses [1] to now also include states with the same quantum numbers, in this case the $\Sigma^{0}$ and $\Lambda^{0}$, and their isospin mixing. This gives a complete description of the $S U(3)$ flavor symmetry expansion of the (baryon) octet. As an example we have numerically investigated $\Sigma^{0}-\Lambda^{0}$ mixing. While the mass splitting is reasonably well determined, to determine reliably the mixing angle will require a better determination of the $S U(3)$ symmetry flavor breaking expansion. An accurate determination of the mixing angle may be useful in baryonic semileptonic decays; see Sec. V. Further work in these directions is in progress.

\section{ACKNOWLEDGMENTS}

The numerical configuration generation (using the BQCD lattice QCD program [18]) and data analysis (using the Chroma software library [19]) was carried out on the IBM BlueGene/Q using DIRAC 2 resources (EPCC, Edinburgh, United Kingdom), the BlueGene/P and $\mathrm{Q}$ at NIC (Jülich, Germany), the SGI ICE 8200 and Cray XC30 at HLRN (The North-German Supercomputer Alliance) and on the NCI National Facility in Canberra, Australia (supported by the Australian Commonwealth Government). This investigation has been supported partly by the EU Grants No. 227431 (Hadron Physics2) and No. 283826 (Hadron Physics3). J. N. was partially supported by EU Grant No. 228398 (HPC-EUROPA2). H. P. is supported by
DFG Grant No. SCHI 422/9-1. J. M.Z. is supported by the Australian Research Council Grant No. FT100100005 and No. DP140103067. We thank all funding agencies.

\section{APPENDIX A: MASS MATRIX SYMMETRIES-AN EXAMPLE}

To illustrate the transformations of the hadron mass matrices with an explicit example, let us write out in full the symmetry matrices for the transformation $u \leftrightarrow d$. A $3 \times 3$ $S U$ (3) matrix which exchanges the $u$ and $d$ quarks in the quark mass matrix Eq. (8) is [see [12], Eq. (128)]

$U=\exp \left\{i \frac{\pi}{2}\left(\lambda_{1}+\sqrt{3} \lambda_{8}\right)\right\}=\left(\begin{array}{ccc}0 & -1 & 0 \\ -1 & 0 & 0 \\ 0 & 0 & -1\end{array}\right)$

[The minus signs ensure that $|U|=1$, as required for an $S U(3)$ matrix.] If we act with this $U$ on the quark mass matrix it simply swaps the $u$ and $d$ quark masses.

$$
U\left(\begin{array}{ccc}
m_{u} & 0 & 0 \\
0 & m_{d} & 0 \\
0 & 0 & m_{s}
\end{array}\right) U^{\dagger}=\left(\begin{array}{ccc}
m_{d} & 0 & 0 \\
0 & m_{u} & 0 \\
0 & 0 & m_{s}
\end{array}\right)
$$

To transform the baryon mass matrix we need an $8 \times 8$ matrix corresponding to Eq. (A1),

$$
\begin{aligned}
U & =\exp \left\{i \frac{\pi}{2}\left(\lambda_{1}+\sqrt{3} \lambda_{8}\right)\right\} \\
& =\left(\begin{array}{ccccccccc}
0 & 1 & 0 & 0 & 0 & 0 & 0 & 0 \\
1 & 0 & 0 & 0 & 0 & 0 & 0 & 0 \\
0 & 0 & 0 & 0 & 0 & 1 & 0 & 0 \\
0 & 0 & 0 & -1 & 0 & 0 & 0 & 0 \\
0 & 0 & 0 & 0 & 1 & 0 & 0 & 0 \\
0 & 0 & 1 & 0 & 0 & 0 & 0 & 0 \\
0 & 0 & 0 & 0 & 0 & 0 & 0 & 1 \\
0 & 0 & 0 & 0 & 0 & 0 & 1 & 0
\end{array}\right),
\end{aligned}
$$

found by using an $8 \times 8$ set of $\lambda$ matrices [defined in [12], Eq. (144)].

What happens to the baryon mass matrix when we rotate it with this $U$ ? 


$$
\begin{gathered}
U\left(\begin{array}{cccccccc}
M_{n}^{2} & 0 & 0 & 0 & 0 & 0 & 0 & 0 \\
0 & M_{p}^{2} & 0 & 0 & 0 & 0 & 0 & 0 \\
0 & 0 & M_{\Sigma^{-}}^{2} & 0 & 0 & 0 & 0 & 0 \\
0 & 0 & 0 & M_{\Sigma \Sigma}^{2} & M_{\Sigma \Lambda}^{2} & 0 & 0 & 0 \\
0 & 0 & 0 & M_{\Lambda \Sigma}^{2} & M_{\Lambda \Lambda}^{2} & 0 & 0 & 0 \\
0 & 0 & 0 & 0 & 0 & M_{\Sigma^{+}}^{2} & 0 & 0 \\
0 & 0 & 0 & 0 & 0 & 0 & M_{\Xi^{-}}^{2} & 0 \\
0 & 0 & 0 & 0 & 0 & 0 & 0 & M_{\Xi^{0}}^{2}
\end{array}\right) U^{\dagger} \\
=\left(\begin{array}{cccccccc}
M_{p}^{2} & 0 & 0 & 0 & 0 & 0 & 0 & 0 \\
0 & M_{n}^{2} & 0 & 0 & 0 & 0 & 0 & 0 \\
0 & 0 & M_{\Sigma^{+}}^{2} & 0 & 0 & 0 & 0 & 0 \\
0 & 0 & 0 & M_{\Sigma \Sigma}^{2} & -M_{\Sigma_{\Lambda}}^{2} & 0 & 0 & 0 \\
0 & 0 & 0 & -M_{\Lambda \Sigma}^{2} & M_{\Lambda \Lambda}^{2} & 0 & 0 & 0 \\
0 & 0 & 0 & 0 & 0 & M_{\Sigma^{-}}^{2} & 0 & 0 \\
0 & 0 & 0 & 0 & 0 & 0 & M_{\Xi^{0}}^{2} & 0 \\
0 & 0 & 0 & 0 & 0 & 0 & 0 & M_{\Xi^{-}}^{2}
\end{array}\right) .
\end{gathered}
$$

The $n$ and $p$ switch masses, as do the $\Sigma^{-}$and $\Sigma^{+}$and the $\Xi^{0}$ and $\Xi^{-}$, all as expected when $u \leftrightarrow d$. In the central block, which tells us about the $\Sigma^{0} \Lambda^{0}$ sector, we see that the diagonal entries are unchanged; the off-diagonal entries have their sign flipped. This is just what should happen under $u \leftrightarrow d$; the eigenvalues (masses of the two states) will be the same, but the mixing angle will be reversed, $\theta_{\Sigma \Lambda} \rightarrow-\theta_{\Sigma \Lambda}$.

\section{APPENDIX B: THE OCTET BARYON MASS MATRIX}

\section{The outer octet baryon masses}

Here we discuss the mass matrix for partially quenched octet baryons in more detail than we could in the body of the paper. The arguments given here are similar to those given in Sec. B.4 of [12] for the meson mass matrix, and in Sec. IV A for the partially quenched decuplet mass formula.

If we have a diagonal quark mass matrix, strangeness, "upness" and "downness" are all conserved quantum numbers. There are therefore only ten nonzero entries in the $8 \times 8$ octet mass matrix, namely the eight diagonal entries, and the two entries corresponding to $\Sigma-\Lambda$ mixing. $\Sigma-\Lambda$ mixing is permitted because both baryons have the same flavor content $(u d s)$; any other mixing would violate flavor conservation.

Since there are ten nonzero entries, we can express the mass matrix in terms of a basis of ten $8 \times 8$ matrices. In [12] we classified these ten matrices according to their symmetries; see Table I. Seven of the matrices are diagonal; they can be read off directly from the table. The table also contains three matrices which mix the $\Sigma$ and $\Lambda$.

In [12] we did not specify the mixing, as we were concentrating on the case of unbroken isospin symmetry, where there is no mixing. We now list the basis matrices $N_{i}$ in full, including the three nondiagonal matrices.

$$
\begin{aligned}
& N_{1}=\left(\begin{array}{cccccccc}
1 & 0 & 0 & 0 & 0 & 0 & 0 & 0 \\
0 & 1 & 0 & 0 & 0 & 0 & 0 & 0 \\
0 & 0 & 1 & 0 & 0 & 0 & 0 & 0 \\
0 & 0 & 0 & 1 & 0 & 0 & 0 & 0 \\
0 & 0 & 0 & 0 & 1 & 0 & 0 & 0 \\
0 & 0 & 0 & 0 & 0 & 1 & 0 & 0 \\
0 & 0 & 0 & 0 & 0 & 0 & 1 & 0 \\
0 & 0 & 0 & 0 & 0 & 0 & 0 & 1
\end{array}\right) \quad N_{2}=\left(\begin{array}{cccccccc}
-1 & 0 & 0 & 0 & 0 & 0 & 0 & 0 \\
0 & -1 & 0 & 0 & 0 & 0 & 0 & 0 \\
0 & 0 & 0 & 0 & 0 & 0 & 0 & 0 \\
0 & 0 & 0 & 0 & 0 & 0 & 0 & 0 \\
0 & 0 & 0 & 0 & 0 & 0 & 0 & 0 \\
0 & 0 & 0 & 0 & 0 & 0 & 0 & 0 \\
0 & 0 & 0 & 0 & 0 & 0 & 1 & 0 \\
0 & 0 & 0 & 0 & 0 & 0 & 0 & 1
\end{array}\right) \quad N_{3}=\left(\begin{array}{cccccccc}
-1 & 0 & 0 & 0 & 0 & 0 & 0 & 0 \\
0 & 1 & 0 & 0 & 0 & 0 & 0 & 0 \\
0 & 0 & -2 & 0 & 0 & 0 & 0 & 0 \\
0 & 0 & 0 & 0 & 0 & 0 & 0 & 0 \\
0 & 0 & 0 & 0 & 0 & 0 & 0 & 0 \\
0 & 0 & 0 & 0 & 0 & 2 & 0 & 0 \\
0 & 0 & 0 & 0 & 0 & 0 & -1 & 0 \\
0 & 0 & 0 & 0 & 0 & 0 & 0 & 1
\end{array}\right) \\
& N_{4}=\left(\begin{array}{cccccccc}
1 & 0 & 0 & 0 & 0 & 0 & 0 & 0 \\
0 & 1 & 0 & 0 & 0 & 0 & 0 & 0 \\
0 & 0 & -2 & 0 & 0 & 0 & 0 & 0 \\
0 & 0 & 0 & -2 & 0 & 0 & 0 & 0 \\
0 & 0 & 0 & 0 & 2 & 0 & 0 & 0 \\
0 & 0 & 0 & 0 & 0 & -2 & 0 & 0 \\
0 & 0 & 0 & 0 & 0 & 0 & 1 & 0 \\
0 & 0 & 0 & 0 & 0 & 0 & 0 & 1
\end{array}\right) \quad N_{5}=\left(\begin{array}{cccccccc}
-1 & 0 & 0 & 0 & 0 & 0 & 0 & 0 \\
0 & 1 & 0 & 0 & 0 & 0 & 0 & 0 \\
0 & 0 & 0 & 0 & 0 & 0 & 0 & 0 \\
0 & 0 & 0 & 0 & \frac{2}{\sqrt{3}} & 0 & 0 & 0 \\
0 & 0 & 0 & \frac{2}{\sqrt{3}} & 0 & 0 & 0 & 0 \\
0 & 0 & 0 & 0 & 0 & 0 & 0 & 0 \\
0 & 0 & 0 & 0 & 0 & 0 & 1 & 0 \\
0 & 0 & 0 & 0 & 0 & 0 & 0 & -1
\end{array}\right) \quad N_{6}=\left(\begin{array}{cccccccc}
1 & 0 & 0 & 0 & 0 & 0 & 0 & 0 \\
0 & 1 & 0 & 0 & 0 & 0 & 0 & 0 \\
0 & 0 & 1 & 0 & 0 & 0 & 0 & 0 \\
0 & 0 & 0 & -3 & 0 & 0 & 0 & 0 \\
0 & 0 & 0 & 0 & -3 & 0 & 0 & 0 \\
0 & 0 & 0 & 0 & 0 & 1 & 0 & 0 \\
0 & 0 & 0 & 0 & 0 & 0 & 1 & 0 \\
0 & 0 & 0 & 0 & 0 & 0 & 0 & 1
\end{array}\right)
\end{aligned}
$$




$$
N_{7}=\left(\begin{array}{cccccccc}
1 & 0 & 0 & 0 & 0 & 0 & 0 & 0 \\
0 & 1 & 0 & 0 & 0 & 0 & 0 & 0 \\
0 & 0 & -2 & 0 & 0 & 0 & 0 & 0 \\
0 & 0 & 0 & 3 & 0 & 0 & 0 & 0 \\
0 & 0 & 0 & 0 & -3 & 0 & 0 & 0 \\
0 & 0 & 0 & 0 & 0 & -2 & 0 & 0 \\
0 & 0 & 0 & 0 & 0 & 0 & 1 & 0 \\
0 & 0 & 0 & 0 & 0 & 0 & 0 & 1
\end{array}\right) \quad N_{8}=\left(\begin{array}{cccccccc}
-1 & 0 & 0 & 0 & 0 & 0 & 0 & 0 \\
0 & 1 & 0 & 0 & 0 & 0 & 0 & 0 \\
0 & 0 & 0 & 0 & 0 & 0 & 0 & 0 \\
0 & 0 & 0 & 0 & -\sqrt{3} & 0 & 0 & 0 \\
0 & 0 & 0 & -\sqrt{3} & 0 & 0 & 0 & 0 \\
0 & 0 & 0 & 0 & 0 & 0 & 0 & 0 \\
0 & 0 & 0 & 0 & 0 & 0 & 1 & 0 \\
0 & 0 & 0 & 0 & 0 & 0 & 0 & -1
\end{array}\right)
$$

These matrices are orthogonal, in the sense that $\operatorname{Tr}\left[N_{i} N_{j}\right]=$ 0 if $i \neq j$.

We can write the (mass matrix) $)^{2}$ in terms of the basis matrices

$$
M^{2}=\sum_{i} K_{i} N_{i}
$$

This expansion is completely general. The coefficients $K_{i}$ could be functions of the pseudoscalar meson masses if we are doing chiral perturbation theory, but in our case we use polynomials of the bare quark masses. The symmetries of the coefficients must match the symmetries of the $N_{i}$ matrices, for example if the matrix has symmetry $A_{1}$ or $E^{+}$its coefficient must be even under $m_{u} \leftrightarrow m_{d}$; if it has symmetry $A_{2}$ or $E^{-}$it must be odd under this interchange.

We find the coefficients $K_{i}$ by making all possible $S U(3)$ rotations on the quark mass matrix, and asking Mathematica to find the most general coefficients $K_{i}$ which lead to a (mass matrix $)^{2}$ which transforms like Eq. (11). Once we know the $K_{i}$ we can then read off the individual baryon masses.

At first order in $\delta \mu_{q}$ we are only allowed singlet and octet matrices, so our $M^{2}$ matrix has to have the form

$$
M^{2}=\sum_{i=1}^{5} K_{i} N_{i}
$$

with only five terms.
When we put this in the computer, we find that at first order, partially quenched, the most general form of the $K_{i}$ consistent with Eq. (11) is

$$
\begin{aligned}
& K_{1}=M_{0}^{2}+a_{1}\left(\delta \mu_{u}+\delta \mu_{d}+\delta \mu_{s}\right) \\
& K_{2}=a_{8_{a}}\left(2 \delta \mu_{s}-\delta \mu_{u}-\delta \mu_{d}\right) \\
& K_{3}=a_{8_{a}}\left(\delta \mu_{u}-\delta \mu_{d}\right) \\
& K_{4}=a_{8_{b}}\left(2 \delta \mu_{s}-\delta \mu_{u}-\delta \mu_{d}\right) \\
& K_{5}=3 a_{8_{b}}\left(\delta \mu_{u}-\delta \mu_{d}\right) .
\end{aligned}
$$

Much of this could be anticipated on general grounds. The form of the polynomials can be read off from Table II. Since $N_{2}$ and $N_{3}$ are part of the same representation, we know that $K_{2}$ and $K_{3}$ are not independent; they must both be proportional to the same coefficient. Likewise, $K_{4}$ and $K_{5}$ must share a coefficient. The only slightly nontrivial features in Eq. (B4) are the proportionality factors relating $K_{3}$ and $K_{5}$ to $K_{2}$ and $K_{4}$, (factors of 1 and 3). These have to be found by considering a symmetry operation that mixes $N_{2}$ with $N_{3}$, and $N_{4}$ with $N_{5}$. Examples of such operations are the interchanges $\delta \mu_{d} \leftrightarrow \delta \mu_{s}$ or $\delta \mu_{u} \leftrightarrow \delta \mu_{s}$, or the cyclic operation $\delta \mu_{u} \rightarrow \delta \mu_{d} \rightarrow \delta \mu_{s} \rightarrow \delta \mu_{u}$.

We are not quite finished; there is one extra constraint coming from partial quenching. If we calculate the neutron mass from Eq. (B4) we have 


$$
\begin{aligned}
M_{n}^{2}= & \left(M^{2}\right)_{11}=K_{1}-K_{2}-K_{3}+K_{4}-K_{5} \\
= & M_{0}^{2}+\delta \mu_{u}\left(a_{1}-4 a_{8_{b}}\right)+\delta \mu_{d}\left(a_{1}+2 a_{8_{a}}+2 a_{8_{b}}\right) \\
& +\delta \mu_{s}\left(a_{1}-2 a_{8_{a}}+2 a_{8_{b}}\right) .
\end{aligned}
$$

However, we know that although the neutron can depend in a symmetric manner on all the sea quark masses, there is no way it can have any information about the mass of the partially quenched valence $s$ quark, so the final term should not occur. We remove this unwanted term by imposing the constraint

$$
a_{1}-2 a_{8_{a}}+2 a_{8_{b}}=0
$$

leaving

$$
M_{n}^{2}=M_{0}^{2}+a_{1}\left(2 \delta \mu_{d}+\delta \mu_{u}\right)-4 a_{8_{b}}\left(\delta \mu_{u}-\delta \mu_{d}\right) .
$$

Finally, we define new parameters

$$
A_{1} \equiv a_{1}, \quad A_{2} \equiv-4 a_{8_{b}},
$$

simply to tidy up the result,

$$
M_{n}^{2}=M_{0}^{2}+A_{1}\left(2 \delta \mu_{d}+\delta \mu_{u}\right)+A_{2}\left(\delta \mu_{u}-\delta \mu_{d}\right) .
$$

The constraint Eq. (B6) does not only remove the unphysical term from the neutron mass formula; it automatically does the same for all the outer baryons, giving them all a mass formula independent of the absent valence quark mass:

$M^{2}(a a b)=M_{0}^{2}+A_{1}\left(2 \delta \mu_{a}+\delta \mu_{b}\right)+A_{2}\left(\delta \mu_{b}-\delta \mu_{a}\right)$.
In terms of the new parameters Eq. (B8) the final expressions for the $K_{i}$ are

$$
\begin{aligned}
K_{1} & =M_{0}^{2}+A_{1}\left(\delta \mu_{u}+\delta \mu_{d}+\delta \mu_{s}\right) \\
K_{2} & =\frac{1}{4}\left(2 A_{1}-A_{2}\right)\left(2 \delta \mu_{s}-\delta \mu_{u}-\delta \mu_{d}\right) \\
K_{3} & =\frac{1}{4}\left(2 A_{1}-A_{2}\right)\left(\delta \mu_{u}-\delta \mu_{d}\right) \\
K_{4} & =-\frac{1}{4} A_{2}\left(2 \delta \mu_{s}-\delta \mu_{u}-\delta \mu_{d}\right) \\
K_{5} & =-\frac{3}{4} A_{2}\left(\delta \mu_{u}-\delta \mu_{d}\right) .
\end{aligned}
$$

At higher order we proceed in the same way, finding the analogue of Eq. (B4) by considering all possible rotations of the quark matrix, and then the analogue of Eq. (B11) by imposing the partially quenched constraint that the absent valence quark cannot appear in the mass formula for $M^{2}(a a b)$. Of course at higher order more of the $K_{i}$ appear; at quadratic order the 27-plet enters, and we need $K_{1}$ to $K_{8}$; at cubic or higher order, all ten $K_{i}$ appear. Also, the expressions for each $K_{i}$ coefficient become longer, as can be seen from Table II. It would be difficult to carry out the calculation by hand, but with the help of a computer we can find all the $K_{i}$, and thus the complete $M^{2}$ matrix.

\section{The $\Sigma-\Lambda$ mass matrix}

In this paper we are primarily interested in the $\Sigma-\Lambda$ sector. Let us concentrate on the $2 \times 2$ block of $M^{2}$ responsible for these two central baryons. From Eq. (B1) we read off

$$
\left(\begin{array}{ll}
M_{\Sigma \Sigma}^{2} & M_{\Sigma \Lambda}^{2} \\
M_{\Lambda \Sigma}^{2} & M_{\Lambda \Lambda}^{2}
\end{array}\right)=\left(\begin{array}{cc}
K_{1}-2 K_{4}-3 K_{6}+3 K_{7} & \frac{2}{\sqrt{3}} K_{5}-\sqrt{3} K_{8}-i K_{10} \\
\frac{2}{\sqrt{3}} K_{5}-\sqrt{3} K_{8}+i K_{10} & K_{1}+2 K_{4}-3 K_{6}-3 K_{7}
\end{array}\right) .
$$

A tidier way to write this is to split the matrix up according to the behavior of the various terms under the permutation group, Eq. (17),

$$
\left(\begin{array}{ll}
M_{\Sigma \Sigma}^{2} & M_{\Sigma \Lambda}^{2} \\
M_{\Lambda \Sigma}^{2} & M_{\Lambda \Lambda}^{2}
\end{array}\right)=P_{A_{1}}\left(\begin{array}{ll}
1 & 0 \\
0 & 1
\end{array}\right)+P_{E^{+}}\left(\begin{array}{cc}
1 & 0 \\
0 & -1
\end{array}\right)+P_{E^{-}}\left(\begin{array}{ll}
0 & 1 \\
1 & 0
\end{array}\right)+P_{A_{2}}\left(\begin{array}{cc}
0 & -i \\
i & 0
\end{array}\right),
$$

where $P_{G}$ means a function of the quark masses with the symmetry $G$ under the $S_{3}$ permutation group. The individual terms in this expansion are given by

$$
P_{A_{1}}=K_{1}-3 K_{6} \quad P_{E^{+}}=-2 K_{4}+3 K_{7} \quad P_{E^{-}}=\frac{2}{\sqrt{3}} K_{5}-\sqrt{3} K_{8} \quad P_{A_{2}}=K_{10} .
$$

In the main part of the paper we give the full cubic expression for the $P_{G}$, derived from the full results for the $K_{i}$. 


\section{APPENDIX C: SCALE INDEPENDENT QUANTITIES TO NNLO}

For completeness we list here the results of Sec. IV to NNLO.

$$
\begin{aligned}
X_{N}^{2}=M_{0}^{2}+\frac{1}{6}\left(B_{0}+B_{1}+B_{3}\right)\left(\delta m_{u}^{2}+\delta m_{d}^{2}+\delta m_{s}^{2}\right)+\left(C_{0}-C_{3}+3 C_{5}\right) \delta m_{u} \delta m_{d} \delta m_{s} \\
C_{0} \rightarrow \tilde{C}_{0}=\frac{C_{3}-3 C_{5}}{M_{0}^{2}}=\tilde{C}_{3}-3 \tilde{C}_{5} \\
\tilde{P}_{A_{1}}=1+3 \tilde{A}_{1} \delta \bar{\mu}+\frac{1}{6} \tilde{B}_{0}\left(\delta m_{u}^{2}+\delta m_{d}^{2}+\delta m_{s}^{2}\right)+\tilde{B}_{1}\left(\delta \mu_{a}^{2}+\delta \mu_{b}^{2}+\delta \mu_{c}^{2}\right) \\
+\frac{1}{4}\left(\tilde{B}_{3}+\tilde{B}_{4}\right)\left[\left(\delta \mu_{c}-\delta \mu_{a}\right)^{2}+\left(\delta \mu_{c}-\delta \mu_{b}\right)^{2}+\left(\delta \mu_{a}-\delta \mu_{b}\right)^{2}\right]+\tilde{C}_{0} \delta m_{u} \delta m_{d} \delta m_{s}+3 \tilde{C}_{1} \delta \bar{\mu}\left(\delta m_{u}^{2}+\delta m_{d}^{2}+\delta m_{s}^{2}\right) \\
-4\left(\tilde{C}_{5}+\tilde{C}_{7}\right) \delta \mu_{a} \delta \mu_{b} \delta \mu_{c}+\frac{1}{2} \tilde{Q}_{1}\left(\delta \mu_{c}+\delta \mu_{a}\right)\left(\delta \mu_{c}+\delta \mu_{b}\right)\left(\delta \mu_{a}+\delta \mu_{b}\right)+\frac{27}{4} \tilde{Q}_{2}\left(\delta \mu_{c}-\delta \bar{\mu}\right)\left(\delta \mu_{a}-\delta \bar{\mu}\right)\left(\delta \mu_{b}-\delta \bar{\mu}\right) \\
\tilde{P}_{E^{+}}=\frac{3}{2} \tilde{A}_{2}\left(\delta \mu_{c}-\delta \bar{\mu}\right)+\frac{1}{2} \tilde{B}_{2}\left(2 \delta \mu_{c}^{2}-\delta \mu_{a}^{2}-\delta \mu_{b}^{2}\right)+\frac{1}{4}\left(\tilde{B}_{3}-\tilde{B}_{4}\right)\left[\left(\delta \mu_{c}-\delta \mu_{a}\right)^{2}+\left(\delta \mu_{c}-\delta \mu_{b}\right)^{2}-2\left(\delta \mu_{a}-\delta \mu_{b}\right)^{2}\right] \\
+\frac{3}{2} \tilde{C}_{2}\left(\delta \mu_{c}-\delta \bar{\mu}\right)\left(\delta m_{u}^{2}+\delta m_{d}^{2}+\delta m_{s}^{2}\right)+6\left(\tilde{C}_{3}-\tilde{C}_{4}\right)\left(\delta \mu_{c}-\delta \bar{\mu}\right) \delta \bar{\mu}^{2} \\
+\frac{1}{6} \tilde{Q}_{3}\left[\left(\delta \mu_{c}-\delta \mu_{a}\right)^{2}+\left(\delta \mu_{c}-\delta \mu_{b}\right)^{2}-2\left(\delta \mu_{a}-\delta \mu_{b}\right)^{2}\right] \delta \bar{\mu}+\frac{1}{8} \tilde{Q}_{4}\left(\delta \mu_{c}-\delta \bar{\mu}\right)\left(\delta \mu_{a}^{2}+\delta \mu_{b}^{2}+\delta \mu_{c}^{2}-3 \delta \bar{\mu}^{2}\right) \\
\quad \frac{\sqrt{3}}{2} \tilde{A}_{2}\left(\delta \mu_{b}-\delta \mu_{a}\right)+\frac{\sqrt{3}}{2} \tilde{B}_{2}\left(\delta \mu_{b}^{2}-\delta \mu_{a}^{2}\right)+\frac{\sqrt{3}}{4}\left(\tilde{B}_{3}-\tilde{B}_{4}\right)\left[\left(\delta \mu_{c}-\delta \mu_{b}\right)^{2}-\left(\delta \mu_{c}-\delta \mu_{a}\right)^{2}\right] \\
+\frac{\sqrt{3}}{2} \tilde{C}_{2}\left(\delta \mu_{b}-\delta \mu_{a}\right)\left(\delta m_{u}^{2}+\delta m_{d}^{2}+\delta m_{s}^{2}\right)+2 \sqrt{3}\left(\tilde{C}_{3}-\tilde{C}_{4}\right)\left(\delta \mu_{b}-\delta \mu_{a}\right) \delta \bar{\mu}^{2} \\
+\frac{1}{8 \sqrt{3}} \tilde{Q} \tilde{Q}_{4}\left(\delta \mu_{b}-\delta \mu_{a}\right)\left(\delta \mu_{a}^{2}+\delta \mu_{b}^{2}+\delta \mu_{c}^{2}-3 \delta \bar{\mu}^{2}\right)-\frac{\sqrt{3}}{2} \tilde{Q}_{3}\left(\delta \mu_{b}-\delta \mu_{a}\right)\left(\delta \mu_{c}-\delta \bar{\mu}\right) \delta \bar{\mu}, \\
\tilde{P}_{A_{2}=} \tilde{C}_{9}\left(\delta \mu_{c}-\delta \mu_{a}\right)\left(\delta \mu_{c}-\delta \mu_{b}\right)\left(\delta \mu_{a}-\delta \mu_{b}\right)
\end{aligned}
$$

where

$$
\begin{aligned}
& \tilde{Q}_{1} \equiv 2 \tilde{C}_{3}+\tilde{C}_{5}+\tilde{C}_{7} \quad \tilde{Q}_{2} \equiv \tilde{C}_{5}-\tilde{C}_{6}+\tilde{C}_{7}+\tilde{C}_{8} \\
& \tilde{Q}_{3} \equiv 4\left(\tilde{C}_{3}-\tilde{C}_{4}\right)+3\left(\tilde{C}_{5}-\tilde{C}_{7}\right) \quad \tilde{Q}_{4} \equiv 2\left(\tilde{C}_{3}-\tilde{C}_{4}\right)+3\left(\tilde{C}_{5}-\tilde{C}_{7}\right)-9\left(\tilde{C}_{6}+\tilde{C}_{8}\right),
\end{aligned}
$$

and $\delta \bar{\mu} \equiv \frac{1}{3}\left(\delta \mu_{a}+\delta \mu_{b}+\delta \mu_{c}\right)$.

$$
\begin{aligned}
\tilde{M}_{\Sigma}^{2}(a a b)= & 1+\tilde{A}_{1}\left(2 \delta \mu_{a}+\delta \mu_{b}\right)+\tilde{A}_{2}\left(\delta \mu_{b}-\delta \mu_{a}\right)+\tilde{B}_{0} \delta m_{l}^{2}+\tilde{B}_{1}\left(2 \delta \mu_{a}^{2}+\delta \mu_{b}^{2}\right)+\tilde{B}_{2}\left(\delta \mu_{b}^{2}-\delta \mu_{a}^{2}\right)+\tilde{B}_{3}\left(\delta \mu_{b}-\delta \mu_{a}\right)^{2} \\
& -2 \tilde{C}_{0} \delta m_{l}^{3}+6\left[\tilde{C}_{1}\left(2 \delta \mu_{a}+\delta \mu_{b}\right)+\tilde{C}_{2}\left(\delta \mu_{b}-\delta \mu_{a}\right)\right] \delta m_{l}^{2}+\tilde{C}_{3}\left(\delta \mu_{a}+\delta \mu_{b}\right)^{3}+\tilde{C}_{4}\left(\delta \mu_{a}+\delta \mu_{b}\right)^{2}\left(\delta \mu_{a}-\delta \mu_{b}\right) \\
& +\tilde{C}_{5}\left(\delta \mu_{a}+\delta \mu_{b}\right)\left(\delta \mu_{a}-\delta \mu_{b}\right)^{2}+\tilde{C}_{6}\left(\delta \mu_{a}-\delta \mu_{b}\right)^{3}
\end{aligned}
$$

and

$$
\begin{aligned}
\tilde{M}_{\Lambda}^{2}\left(a a^{\prime} b\right)= & 1+\tilde{A}_{1}\left(2 \delta \mu_{a}+\delta \mu_{b}\right)-\tilde{A}_{2}\left(\delta \mu_{b}-\delta \mu_{a}\right)+\tilde{B}_{0} \delta m_{l}^{2}+\tilde{B}_{1}\left(2 \delta \mu_{a}^{2}+\delta \mu_{b}^{2}\right)-\tilde{B}_{2}\left(\delta \mu_{b}^{2}-\delta \mu_{a}^{2}\right)+\tilde{B}_{4}\left(\delta \mu_{b}-\delta \mu_{a}\right)^{2} \\
& -2 \tilde{C}_{0} \delta m_{l}^{3}+6\left[\tilde{C}_{1}\left(2 \delta \mu_{a}+\delta \mu_{b}\right)-\tilde{C}_{2}\left(\delta \mu_{b}-\delta \mu_{a}\right)\right] \delta m_{l}^{2}+\tilde{C}_{3}\left(\delta \mu_{a}+\delta \mu_{b}\right)^{3}+\left(\tilde{C}_{4}-2 \tilde{C}_{3}\right)\left(\delta \mu_{a}+\delta \mu_{b}\right)^{2}\left(\delta \mu_{b}-\delta \mu_{a}\right) \\
& +\tilde{C}_{7}\left(\delta \mu_{a}+\delta \mu_{b}\right)\left(\delta \mu_{b}-\delta \mu_{a}\right)^{2}+\tilde{C}_{8}\left(\delta \mu_{b}-\delta \mu_{a}\right)^{3} .
\end{aligned}
$$

Equation (78) is generalized to

$$
D_{\Sigma \Lambda}^{\mathrm{sym}}=\tilde{A}_{2}+\tilde{B}_{2}\left(\delta \mu_{a}+\delta \mu_{b}\right)+6 \tilde{C}_{2} \delta m_{l}^{2}+\left(\tilde{C}_{3}-\tilde{C}_{4}\right)\left(\delta \mu_{a}+\delta \mu_{b}\right)^{2}-\frac{1}{2}\left(\tilde{C}_{6}+\tilde{C}_{8}\right)\left(\delta \mu_{b}-\delta \mu_{a}\right)^{2} .
$$




\section{APPENDIX D: TABLES}

Table VII gives the PQ baryon masses when all three valence quarks are different, while Table VIII gives the masses when two valence quarks are mass degenerate. The three sea quark kappa values are $\kappa_{l}$ (twice) and $\kappa_{s}$, while the valence quark values are $\kappa_{a}, \kappa_{b}, \kappa_{c}$.

TABLE VII. Baryon masses used with valence quark kappa values $\kappa_{a} \neq \kappa_{b} \neq \kappa_{c}$.

\begin{tabular}{lccccccc}
\hline \hline$\kappa_{l}$ & $\kappa_{s}$ & $\kappa_{a}$ & $\kappa_{b}$ & $\kappa_{c}$ & $V$ & $M_{H}(a b c)$ & $M_{L}(a b c)$ \\
\hline 0.120900 & 0.120900 & 0.120900 & 0.120512 & 0.120000 & $32^{3} \times 64$ & $0.5698(31)$ & $0.5580(26)$ \\
0.120900 & 0.120900 & 0.121095 & 0.120512 & 0.120000 & $32^{3} \times 64$ & $0.5577(38)$ & $0.5433(31)$ \\
0.120900 & 0.120900 & 0.121095 & 0.120900 & 0.120000 & $32^{3} \times 64$ & $0.5300(45)$ & $0.5123(39)$ \\
0.120900 & 0.120900 & 0.121095 & 0.120900 & 0.120512 & $32^{3} \times 64$ & $0.4892(48)$ & $0.4777(43)$ \\
0.120900 & 0.120900 & 0.120900 & 0.120000 & 0.118000 & $32^{3} \times 64$ & $0.7278(26)$ & $0.7087(24)$ \\
\hline \hline
\end{tabular}

TABLE VIII. Baryon masses used with valence quark kappa values $\kappa_{a}=\kappa_{b}$.

\begin{tabular}{|c|c|c|c|c|c|c|c|}
\hline$\kappa_{l}$ & $\kappa_{s}$ & $\kappa_{a}$ & $\kappa_{b}$ & $\kappa_{c}$ & V & $M_{\Sigma}(a a b)$ & $M_{\Lambda}\left(a a^{\prime} b\right)$ \\
\hline 0.120900 & 0.120900 & 0.120000 & 0.120000 & 0.118000 & $32^{3} \times 64$ & $0.7789(23)$ & $0.7684(23)$ \\
\hline 0.120900 & 0.120900 & 0.120000 & 0.120000 & 0.120000 & $32^{3} \times 64$ & $0.6588(23)$ & $0.6588(23)$ \\
\hline 0.120900 & 0.120900 & 0.120000 & 0.120000 & 0.120512 & $32^{3} \times 64$ & $0.6232(24)$ & $0.6290(26)$ \\
\hline 0.120900 & 0.120900 & 0.120000 & 0.120000 & 0.120900 & $32^{3} \times 64$ & $0.5945(26)$ & $0.6058(29)$ \\
\hline 0.120900 & 0.120900 & 0.120512 & 0.120512 & 0.120000 & $32^{3} \times 64$ & $0.5945(25)$ & $0.5892(28)$ \\
\hline 0.120900 & 0.120900 & 0.120512 & 0.120512 & 0.120512 & $32^{3} \times 64$ & $0.5564(27)$ & $0.5564(27)$ \\
\hline 0.120900 & 0.120900 & 0.120512 & 0.120512 & 0.120900 & $32^{3} \times 64$ & $0.5247(30)$ & $0.5328(34)$ \\
\hline 0.120900 & 0.120900 & 0.120900 & 0.120900 & 0.116000 & $32^{3} \times 64$ & $0.7811(34)$ & $0.7438(33)$ \\
\hline 0.120900 & 0.120900 & 0.120900 & 0.120900 & 0.118000 & $32^{3} \times 64$ & $0.6739(33)$ & $0.6442(32)$ \\
\hline 0.120900 & 0.120900 & 0.120900 & 0.120900 & 0.120000 & $32^{3} \times 64$ & $0.5435(32)$ & $0.5277(37)$ \\
\hline 0.120900 & 0.120900 & 0.120900 & 0.120900 & 0.120512 & $32^{3} \times 64$ & $0.5031(35)$ & $0.4938(41)$ \\
\hline 0.120900 & 0.120900 & 0.120000 & 0.120000 & 0.121095 & $32^{3} \times 64$ & $0.5806(31)$ & $0.5960(37)$ \\
\hline 0.120900 & 0.120900 & 0.120512 & 0.120512 & 0.121095 & $32^{3} \times 64$ & $0.5086(36)$ & $0.5234(46)$ \\
\hline 0.120900 & 0.120900 & 0.120900 & 0.120900 & 0.121095 & $32^{3} \times 64$ & $0.4502(55)$ & $0.4606(80)$ \\
\hline 0.120900 & 0.120900 & 0.121095 & 0.121095 & 0.120000 & $32^{3} \times 64$ & $0.5226(65)$ & $0.5029(123)$ \\
\hline 0.120900 & 0.120900 & 0.121095 & 0.121095 & 0.120512 & $32^{3} \times 64$ & $0.4838(77)$ & $0.4744(177)$ \\
\hline 0.120900 & 0.120900 & 0.121095 & 0.121095 & 0.120900 & $32^{3} \times 64$ & $0.4519(42)$ & $0.4613(300)$ \\
\hline 0.121040 & 0.120620 & 0.120620 & 0.120620 & 0.120620 & $32^{3} \times 64$ & $0.5265(16)$ & $0.5265(16)$ \\
\hline 0.121040 & 0.120620 & 0.120620 & 0.120620 & 0.121040 & $32^{3} \times 64$ & $0.4907(21)$ & $0.5014(30)$ \\
\hline 0.121040 & 0.120620 & 0.121040 & 0.121040 & 0.120620 & $32^{3} \times 64$ & $0.4697(33)$ & $0.4547(43)$ \\
\hline 0.121040 & 0.120620 & 0.121040 & 0.121040 & 0.121040 & $32^{3} \times 64$ & $0.4267(50)$ & $0.4267(50)$ \\
\hline 0.121095 & 0.120512 & 0.120512 & 0.120512 & 0.120512 & $32^{3} \times 64$ & $0.5446(16)$ & $0.5446(16)$ \\
\hline 0.121095 & 0.120512 & 0.120512 & 0.120512 & 0.121095 & $32^{3} \times 64$ & $0.4971(21)$ & $0.5054(31)$ \\
\hline 0.121095 & 0.120512 & 0.121095 & 0.121095 & 0.120512 & $32^{3} \times 64$ & $0.4690(37)$ & $0.4510(58)$ \\
\hline 0.121095 & 0.120512 & 0.121095 & 0.121095 & 0.121095 & $32^{3} \times 64$ & $0.4140(61)$ & $0.4140(61)$ \\
\hline 0.121145 & 0.120413 & 0.120413 & 0.120413 & 0.120413 & $32^{3} \times 64$ & $0.5682(13)$ & $0.5682(13)$ \\
\hline 0.121145 & 0.120413 & 0.120413 & 0.120413 & 0.121145 & $32^{3} \times 64$ & $0.5092(19)$ & $0.5239(23)$ \\
\hline 0.121145 & 0.120413 & 0.121145 & 0.121145 & 0.120413 & $32^{3} \times 64$ & $0.4761(39)$ & $0.4507(65)$ \\
\hline 0.121145 & 0.120413 & 0.121145 & 0.121145 & 0.121145 & $32^{3} \times 64$ & $0.4016(89)$ & $0.4016(89)$ \\
\hline 0.120900 & 0.120900 & 0.120900 & 0.120900 & 0.120900 & $32^{3} \times 64$ & $0.4673(27)$ & $0.4673(27)$ \\
\hline 0.121166 & 0.120371 & 0.120371 & 0.120371 & 0.120371 & $48^{3} \times 96$ & $0.5730(26)$ & $0.5730(26)$ \\
\hline 0.121166 & 0.120371 & 0.120371 & 0.120371 & 0.121166 & $48^{3} \times 96$ & $0.5083(35)$ & $0.5247(46)$ \\
\hline 0.121166 & 0.120371 & 0.121166 & 0.121166 & 0.120371 & $48^{3} \times 96$ & $0.4680(66)$ & $0.4322(66)$ \\
\hline 0.121166 & 0.120371 & 0.121166 & 0.121166 & 0.121166 & $48^{3} \times 96$ & $0.3817(123)$ & $0.3817(123)$ \\
\hline
\end{tabular}


[1] R. Horsley, J. Najjar, Y. Nakamura, D. Pleiter, P. E. L. Rakow, G. Schierholz, and J. M. Zanotti (QCDSF-UKQCD Collaborations), Phys. Rev. D 86, 114511 (2012).

[2] K. A. Olive et al. (Particle Data Group), Chin. Phys. C 38, 090001 (2014).

[3] N. H. Christ, C. Dawson, T. Izubuchi, C. Jung, Q. Liu, R. D. Mawhinney, C. T. Sachrajda, A. Soni, and R. Zhou (RBC and UKQCD Collaborations), Phys. Rev. Lett. 105, 241601 (2010).

[4] J. J. Dudek, R. G. Edwards, B. Joó, M. J. Peardon, D. G. Richards, and C. E. Thomas (Hadron Spectrum Collaboration), Phys. Rev. D 83, 111502 (2011).

[5] E. B. Gregory, A. C. Irving, C. M. Richards, and C. McNeile (UKQCD Collaboration), Phys. Rev. D 86, 014504 (2012).

[6] C. Michael, K. Ottnad, and C. Urbach (ETM Collaboration), Phys. Rev. Lett. 111, 181602 (2013).

[7] N. Isgur, Phys. Rev. D 21, 779 (1980); 23, 817(E) (1981).

[8] J. Gasser and H. Leutwyler, Phys. Rep. 87, 77 (1982).

[9] S.-L. Zhu, W.-Y. P. Hwang, and Z.-S. Yang, Phys. Rev. D 57, 1524 (1998).

[10] N. Yagisawa, T. Hatsuda, and A. Hayashigaki, Nucl. Phys. A699, 665 (2002).

[11] P. W. Atkins, M. S. Child, and C. S. G. Phillips, Tables for Group Theory (Oxford University Press, Oxford, 1970).
[12] W. Bietenholz, V. Bornyakov, M. Göckeler, R. Horsley, W. G. Lockhart, Y. Nakamura, H. Perlt, D. Pleiter, P. E. L. Rakow, G. Schierholz, A. Schiller, T. Streuer, H. Stüben, F. Winter, and J. M. Zanotti (QCDSF-UKQCD Collaboration), Phys. Rev. D 84, 054509 (2011).

[13] S. Coleman and S. L. Glashow, Phys. Rev. Lett. 6, 423 (1961).

[14] G. Karl, Phys. Lett. B 328, 149 (1994); 341, 449(E) (1995).

[15] N. Cundy, M. Göckeler, R. Horsley, T. Kaltenbrunner, A. D. Kennedy, Y. Nakamura, H. Perlt, D. Pleiter, P. E. L. Rakow, A. Schäfer, G. Schierholz, A. Schiller, H. Stüben, and J. M. Zanotti (QCDSF-UKQCD Collaboration), Phys. Rev. D 79, 094507 (2009).

[16] D. Griffiths, Introduction to Elementary Particles (John Wiley, New York, 1987).

[17] Y.-B. Yang, Y. Chen, A. Alexandru, S.-J. Dong, T. Draper, M. Gong, F. X. Lee, A. Li, K.-F. Liu, Z. Liu, and M. Lujan ( $\chi$ QCD Collaboration), arXiv:1410.3343.

[18] Y. Nakamura and H. Stüben, Proc. Sci., LATTICE2010 040, (2010) [arXiv:1011.0199].

[19] R. G. Edwards and B. Joó, Nucl. Phys. B, Proc. Suppl. B140, 832 (2005). 